\title{
Noninvasively improving the orbit-response matrix while continuously correcting the orbit
}

\author{
Ingvar Ziemann $\odot$ \\ KTH, Royal Institute of Technology, 10044 Stockholm, Sweden \\ Volker Ziemann $\odot^{*}$ \\ Uppsala University, 75120 Uppsala, Sweden
}

(Received 13 April 2021; accepted 30 June 2021; published 23 July 2021)

\begin{abstract}
Based on continuously recorded beam positions and corrector excitations from, for example, a closedorbit feedback system we describe an algorithm that continuously updates an estimate of the orbit response matrix. The speed of convergence can be increased by adding very small perturbations, so-called dither, to the corrector excitations. Estimates for the rate of convergence and the asymptotically achievable accuracies are provided.
\end{abstract}

DOI: 10.1103/PhysRevAccelBeams.24.072804

\section{INTRODUCTION}

The orbit-response matrix relates changes of the dipole corrector magnets to orbit changes that are observed on the beam position monitor system. It is of paramount importance for maintaining stable beam positions in storage rings, which is typically accomplished by "slow" orbit correction systems [1-4] and "fast" feedback systems [5,6]. They either use a response matrix generated from a computer model of the accelerator or a measured matrix found by varying one corrector at a time and observing the ensuing changes with the beam position monitor (BPM) system.

As a matter of fact, comparing the measured matrix with a matrix derived from a computer model, as discussed in [7-9], makes it possible to track down deficient hardware, such as incorrectly calibrated power supplies or scale errors of position monitors. Usually, the response matrix is measured in dedicated shifts, labeled "machine development," where the excitation of one corrector after the other is varied and the resulting changes of the positions on the orbit monitor system are recorded, which is commonly referred to as "open loop" measurements. In this report, we discuss an algorithm that complements the existing methods. It requires no dedicated beam time and slowly improves an estimate of the response matrix quasi for free by using information from the "closed loop" orbit feedback system. The procedure, based on a recursive least-squares

\footnotetext{
*volker.ziemann@physics.uu.se
}

Published by the American Physical Society under the terms of the Creative Commons Attribution 4.0 International license. Further distribution of this work must maintain attribution to the author(s) and the published article's title, journal citation, and DOI. algorithm [10,11], is completely noninvasive and can run while operating the accelerator in production mode-producing luminosity in a collider, or photons in a light source. It has the remarkable property that the error bars asymptotically approach zero as the estimated response matrix approaches the "real" response matrix. The algorithm is, however, slow, because it "learns from noise" but might nevertheless prove useful to continuously improve the response matrix at times normally not accessible for machine improvement. This opens the possibility to track down very slow changes of hardware parameters when post-processing the response matrix with, for example, LOCO [8].

This report is organized as follows: in the next section we develop the algorithm, followed by Sec. III, where we introduce a simple model storage ring used to illustrate it. In Sec. IV we introduce dithering as a way to speed up the algorithm, before we explore its convergence properties, both during the early stages in Sec. V, and in the asymptotic regime in Sec. VI. Before concluding, we address a number of technical issues and extensions to the algorithm in Sec. VII.

\section{THE ALGORITHM}

The response matrix $B$ with matrix elements $B^{i j}$ relates the change in excitation $u^{j}$ of steering magnet $j$ with $1 \leq$ $j \leq m$ to a change of the beam position $x^{i}$ with $1 \leq i \leq n$ on monitor $i$. Here superscripts denote different monitors and correctors. We will use the notation from quantum mechanics with bra states denoting column vectors and ket states denoting row vectors, which will prove convenient later on. We thus collectively denote the values of all $n$ BPM by $|x\rangle$ and the $m$ correctors by $|u\rangle$. Correcting the orbit then means to add a perturbation $B|u\rangle$ to the orbit $|x\rangle$ that minimizes the residual orbit $|\tilde{x}\rangle$ after correction. It is given by 


$$
|\tilde{x}\rangle=|x\rangle+B|u\rangle+|w\rangle,
$$

where $|w\rangle$ describes noise in the system, for example, due to ground motion or BPM noise. When correcting the orbit, we have to find corrector excitations $|u\rangle$ that minimize $\langle\tilde{x} \mid \tilde{x}\rangle$. One problem is that we do not have complete knowledge of the system matrix $B$. All we do know is a more or less accurate estimate $\tilde{B}$ that was previously derived from a computer model or from measurements and use that when correcting the orbit.

Assuming that the position monitors report values $|x\rangle$ and furthermore assuming that the desired orbit is centered around zero, allows us to calculate the desired corrector excitations $|u\rangle$ from inverting $\tilde{B}$, the approximation of $B$ from Eq. (1). If $\tilde{B}$ is square $(n=m)$ and invertible this is just the matrix-inverse $-\tilde{B}^{-1}$, where the minus sign ensures that the effect of the correctors cancels the observed orbit. If $\tilde{B}$ is overdetermined $(n>m)$ this is accomplished by the Moore-Penrose pseudoinverse $-\left(\tilde{B}^{\top} \tilde{B}\right)^{-1} \tilde{B}^{\top}$, which follows from minimizing $(|x\rangle+\tilde{B}|u\rangle)^{\top}(|x\rangle+\tilde{B}|u\rangle)$ with respect to $|u\rangle$. If $\tilde{B}$ is underdetermined $(n<m)$ it can be inverted using singular value decomposition. In general, we denote the linear dependence of the corrector excitations on the observed orbit $|x\rangle$ by the "correction" matrix $K$, such that $|u\rangle=-K|x\rangle$.

Our task is now to extract information from repeatedly correcting the orbit and correlating the orbit change with the used corrector changes $|u\rangle$. To this end we note that the noise $|w\rangle$ and the mismatch of the "real" accelerator model $B$ and $\tilde{B}$ from which $K$ is derived, causes the correction to be imperfect. We model this dependence by the dynamical system

$\left|x_{t+1}\right\rangle=\left|x_{t}\right\rangle+B\left|u_{t}\right\rangle+\left|w_{t}\right\rangle \quad$ with $\quad\left|u_{t}\right\rangle=-K\left|x_{t}\right\rangle$,

where the subscript $t$ denotes a discrete time step from one iteration of the orbit correction to the next. We assume that the noise $\left|w_{t}\right\rangle$ is Gaussian and characterized by the expectation value $\mathbf{E}\left\{\left|w_{s}\right\rangle\left\langle w_{t}\right|\right\}=\sigma_{w}^{2} C \delta_{s t}$. Here $\sigma_{w}^{2} C$ is the spatial covariance matrix, where $\sigma_{w}$ is the rms magnitude and $C$ describes correlations among different BPM. In Appendix D we will return to the general case, but assume $C$ to be a $n \times n$ unit matrix in the main text. Furthermore, $\delta_{s t}$ is the Kronecker delta, which implies that we treat noise to be uncorrelated from one iteration to the next. Note also that the effect of power supply noise $\left|\tilde{v}_{t}\right\rangle$ added to $\left|u_{t}\right\rangle$ is equivalent to additional noise on the monitors with magnitude $B\left|\tilde{v}_{t}\right\rangle$. In Eq. (2) we implicitly omit fast timedependent effects, such as latency in the power supplies or the computation chain as well as the effect of eddy currents. In Sec. VII we briefly discuss how to include these effects, but in the main text all transient effects are assumed to have settled to a new equilibrium from one iteration to the next. Now the interpretation of Eq. (2) is straightforward: the system responds with the "real" response matrix $B$ to a change of the corrector excitation by $\left|u_{t}\right\rangle$ that was calculated with the approximative inverse $K$ and the orbit $\left|x_{t}\right\rangle$. At the same time, noise enters the system through $\left|w_{t}\right\rangle$, such that the residual orbit $\left|x_{t+1}\right\rangle$ after the correction is not necessarily equal to zero. Iterating the orbit correction, which is what orbit feedback systems essentially do, can now be modeled by iterating the system described by Eq. (2).

In order to find an estimate $\hat{B}$ of the system matrix $B$ one row-corresponding to a particular BPM $i$-at a time, we construct linear systems of equations for each time step and solve the resulting sequence of equations with a recursive least-squares algorithm $[10,11]$. To set up the equations, for the time being, we ignore the noise $\left|w_{t}\right\rangle$ and formulate Eq. (2) for this BPM as a constraint for $\hat{B}$. Writing the constraints over consecutive readings $x_{s}^{i}$, we find

$$
\begin{aligned}
x_{s+1}^{i}-x_{s}^{i} & =\left(\begin{array}{ll}
\hat{B}^{i 1} & \ldots \hat{B}^{i m}
\end{array}\right)\left(\begin{array}{c}
u_{s}^{1} \\
\vdots \\
u_{s}^{m}
\end{array}\right) \\
& =\left(\begin{array}{lll}
u_{s}^{1} & \ldots & u_{s}^{m}
\end{array}\right)\left(\begin{array}{c}
\hat{B}^{i 1} \\
\vdots \\
\hat{B}^{i m}
\end{array}\right),
\end{aligned}
$$

where the second equality follows from exchanging the order of writing the scalar product of row $i$ of $\hat{B}$ and corrector excitations $u_{s}^{j}$. In the next step we assemble multiple copies of this equation from different times $1 \leq$ $s \leq T$ in the form of a matrix

$$
\begin{aligned}
\left(\begin{array}{c}
x_{2}^{i}-x_{1}^{i} \\
\vdots \\
x_{T+1}^{i}-x_{T}^{i}
\end{array}\right) & =\left(\begin{array}{ccc}
u_{1}^{1} & \ldots & u_{1}^{m} \\
& \vdots & \\
u_{T}^{1} & \ldots & u_{T}^{m}
\end{array}\right)\left(\begin{array}{c}
\hat{B}^{i 1} \\
\vdots \\
\hat{B}^{i m}
\end{array}\right) \\
& =U_{T}\left(\begin{array}{c}
\hat{B}^{i 1} \\
\vdots \\
\hat{B}^{i m}
\end{array}\right)=U_{T}\left|\hat{B}^{i:}\right\rangle
\end{aligned}
$$

and denote the matrix containing the corrector excitations $u_{s}^{j}$ by $U_{T}$, which thus contains the excitations of all correctors stacked one by one on top of the other. Likewise, the vector on the left-hand side contains the orbit differences that each of the steering magnet excitations causes. If we now record BPM positions and corresponding corrector excitations for a long time $T$, the system of equations in Eq. (4) is vastly overdetermined, provided that the noise really affects all possible degrees of freedom of the system, which implies that in general the covariance matrix $C$ must have full rank. Since we assume 
$C$ to be the unit matrix, this is the case and we can solve Eq. (4) in the least-squares sense with the pseudoinverse mentioned above. We find

$$
\left|\hat{B}_{T}^{i:}\right\rangle=\left(\begin{array}{c}
\hat{B}_{T}^{i 1} \\
\vdots \\
\hat{B}_{T}^{i m}
\end{array}\right)=\left(U_{T}^{\top} U_{T}\right)^{-1} U_{T}^{\top}\left(\begin{array}{c}
x_{2}^{i}-x_{1}^{i} \\
\vdots \\
x_{T+1}^{i}-x_{T}^{i}
\end{array}\right),
$$

which provides an estimate for row $i$ of the matrix $\hat{B}_{T}^{i}$ after $T$ iterations of the orbit corrections. Repeating this procedure for all BPMs provides us with an estimate for the complete system matrix $\hat{B}_{T}$.

In passing, we point out that Eq. (5) describes a linear map from the vector with the position differences on the right-hand side onto the vector with row $i$ of $\hat{B}_{T}$, which allows us to calculate an empirical (data-driven) covariance matrix of the $\hat{B}_{T}$ from the covariance matrix of the position difference, which is $2 \sigma_{w}^{2}$ times the unit matrix. The error bars $\sigma(\hat{B})$ of the fitted $\hat{B}_{T}$ are therefore approximately given by the square root of the diagonal elements of $2 \sigma_{w}^{2}\left(U_{T}^{\top} U_{T}\right)^{-1}$, which will prove useful later on.

Calculating the pseudoinverse of $U_{T}$ for more and more iterations becomes numerically very expensive. There is, however, an elegant way of iteratively updating the pseudoinverse using the Sherman-Morrison [12] formula. It is based on updating $P_{T}=\left(U_{T}^{\top} U_{T}\right)^{-1}$ and $\hat{B}_{T}$ as the matrix $U_{T}$ grows one row at a time by adding the row vector $\left\langle u_{T+1}\right|=\left(u_{T+1}^{1}, \ldots, u_{T+1}^{m}\right)$ to it. This entails that we can write $P_{T+1}^{-1}=P_{T}^{-1}+\left|u_{T+1}\right\rangle\left\langle u_{T+1}\right|$. In Appendix A we show that its inverse is given by

$$
P_{T+1}=P_{T}-\frac{P_{T}\left|u_{T+1}\right\rangle\left\langle u_{T+1}\right| P_{T}}{1+\left\langle u_{T+1}\left|P_{T}\right| u_{T+1}\right\rangle} .
$$

With $P_{T+1}=\left(U_{T+1}^{\top} U_{T+1}\right)^{-1}$ known, we can calculate an updated approximation [13] of the response matrix $\hat{B}_{T+1}$ from

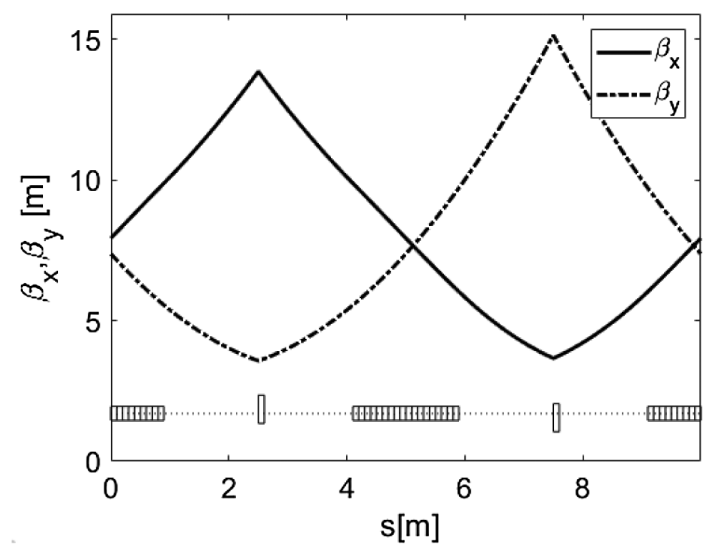

$\hat{B}_{T+1}=\hat{B}_{T}+\frac{\left(\left|x_{T+2}\right\rangle-\left|x_{T+1}\right\rangle-\hat{B}_{T}\left|u_{T+1}\right\rangle\right)\left\langle u_{T+1}\right| P_{T}}{1+\left\langle u_{T+1}\left|P_{T}\right| u_{T+1}\right\rangle}$.

We refer to Appendix B and [13] for the derivation. Note that in Eqs. (6) and (7) the right-hand sides only depend on $P_{T}$ and $\hat{B}_{T}$ from the previous iteration, the new corrector excitations $\left|u_{T+1}\right\rangle$, and the most recent change in the orbit $\left|x_{T+2}\right\rangle-\left|x_{T+1}\right\rangle$. These two equations now allow us to continuously update the response matrix while correcting the orbit with $K$. All we do here is correlating the change of the orbit $\left|x_{T+2}\right\rangle-\left|x_{T+1}\right\rangle$ with the corrector excitations $\left|u_{T+1}\right\rangle$ that cause this change and then update our approximation of $\hat{B}$ in the process.

With the basic algorithm worked out, we simulate its performance in the following section.

\section{SIMULATION}

In order to test the algorithm we prepared response matrices for a small ring consisting of ten FODO cells each having phase advances of $\mu_{x} / 2 \pi=0.228$ and $\mu_{y} / 2 \pi=$ 0.238 in the horizontal and vertical plane respectively. The tunes of the ring therefore are $Q_{x}=2.28$ and $Q_{y}=2.38$. Moreover, there are two 18-degree sector dipole magnets in each cell. The beta functions of one cell are shown on the left-hand side in Fig. 1. We place a corrector and a BPM at the same location as the (thin-lens) focusing quadrupole, which then accounts for ten correctors and ten BPM, each. In order to keep the simulation transparent, we calculate the response matrix $B$ between these correctors and BPM in the horizontal plane only. Most of the simulations are done for equal numbers of correctors and monitors; we address other cases in Sec. VII. The response matrix derived from this unperturbed ring is the "ideal" response matrix $\tilde{B}$ that we use to derive the correction matrix $K=\left(\tilde{B}^{\top} \tilde{B}\right)^{-1} \tilde{B}^{\top}$ to correct the orbit. In order to simplify the theoretical analysis in Sec. VI, in the remainder of this report we confine ourself to a constant correction matrix $\tilde{B}$. If, instead we were to use

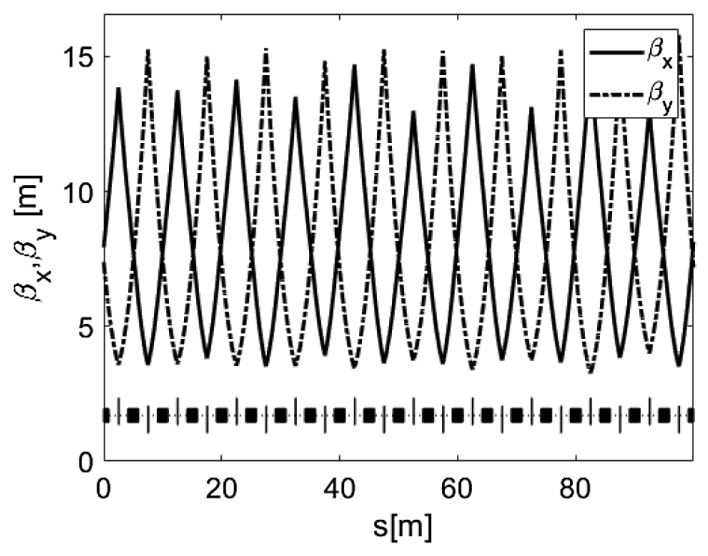

FIG. 1. Left: the beta functions of one cell. Right: the beta functions of the ring where the focal lengths of all quadrupoles are randomly perturbed by $5 \%$ which causes a moderate beating. 
the constantly updating $\hat{B}_{T}$ for the correction, the algorithm would be adaptive. In order to determine the "real" response matrix $B$, we randomly vary the focal lengths of the quadrupoles with a rms of 5\% and recalculate the response matrix $B$ for the perturbed ring. We take notice that the rms magnitude of the response coefficients is $6.6 \mathrm{~m} / \mathrm{rad}$. In order to quantify the estimation error $b_{T}=$ $\hat{B}_{T}-B$ after $T$ iterations, we introduce the rms value of $b_{T}$, calculated over all matrix elements as discrepancy $\left|b_{T}\right|_{r m s}$. It can also be calculated from

$$
\left|b_{T}\right|_{r m s}=\sqrt{\frac{\operatorname{Trace}\left(\left(\hat{B}_{T}-B\right)^{\top}\left(\hat{B}_{T}-B\right)\right)}{n m}} .
$$

Evaluating the initial value $\left|b_{0}\right|_{\text {rms }}$ for our model storage ring, we find that it is approximately $0.3 \mathrm{~m} / \mathrm{rad}$ which accounts for a $5 \%$ rms deviation of the response matrix coefficients. The simulations are based on Matlab scripts that use beam optics functions from [14]. The code illustrating one iteration of the algorithm is reproduced and commented in Appendix C.

Running the simulation for $10^{5}$ iterations, which takes a few seconds on a desktop computer, produces Fig. 2, which shows the evolution of the rms orbit $\sigma_{x}^{2}=\sqrt{\langle x \mid x\rangle}$ and the discrepancy $\left|b_{T}\right|_{\text {rms }}$ between the "real" and the estimated response matrix. We initialized the estimate for $\hat{B}_{0}$ with the response matrix for the ring without quadrupole gradient errors and $P_{0}$ with the unit matrix. The rms amplitude of the noise $\sigma_{w}$ was chosen to be $0.1 \mathrm{~mm}$. Note that the upper plot, which shows the rms orbit $\sigma_{x}$ for the duration of the simulation clearly verifies this; the mean is close to $0.1 \mathrm{~mm}$. At the same time, the discrepancy $\left|b_{T}\right|_{r m s}$, shown on the lower plot, is approximately halved to a final value $\left|b_{f}\right|_{\text {rms }}=0.168 \mathrm{~m} / \mathrm{rad}$, which shows that the algorithm works.

Repeating the same simulation (always for $10^{5}$ iterations) for different values of $\sigma_{w}$ and recording the final discrepancy $\left|b_{f}\right|_{r m s}$ produces the plot shown in Fig. 3. Here we find that increasing noise levels are beneficial for the rate of convergence, up to about $\sigma_{w}=0.5 \mathrm{~mm}$, where the induced changes in $b$ during one iteration become comparable to the magnitude of $b$. We need to stress that the plotted values are those reached after $10^{5}$ iterations. They are not the asymptotic levels.

The algorithm is rather stable. We ran simulations where we initialized $\hat{B}$ with random matrices or other made-up starting guesses. The algorithm, after an initial transient period, always converged toward the "real" matrix $B$.

We point out that the convergence depends on the noise level, where more noise moves the correctors around more and actually improves the convergence, but the rate is still rather slow, on the order of several $10^{5}$ iterations, which would correspond to about three hours real time, provided that the feedback operates at an update rate of 10 per second. Moreover, the asymptotically achievable discrepancy is of considerable interest. We will address these topics below after having introduced the effect of additional corrector perturbations.

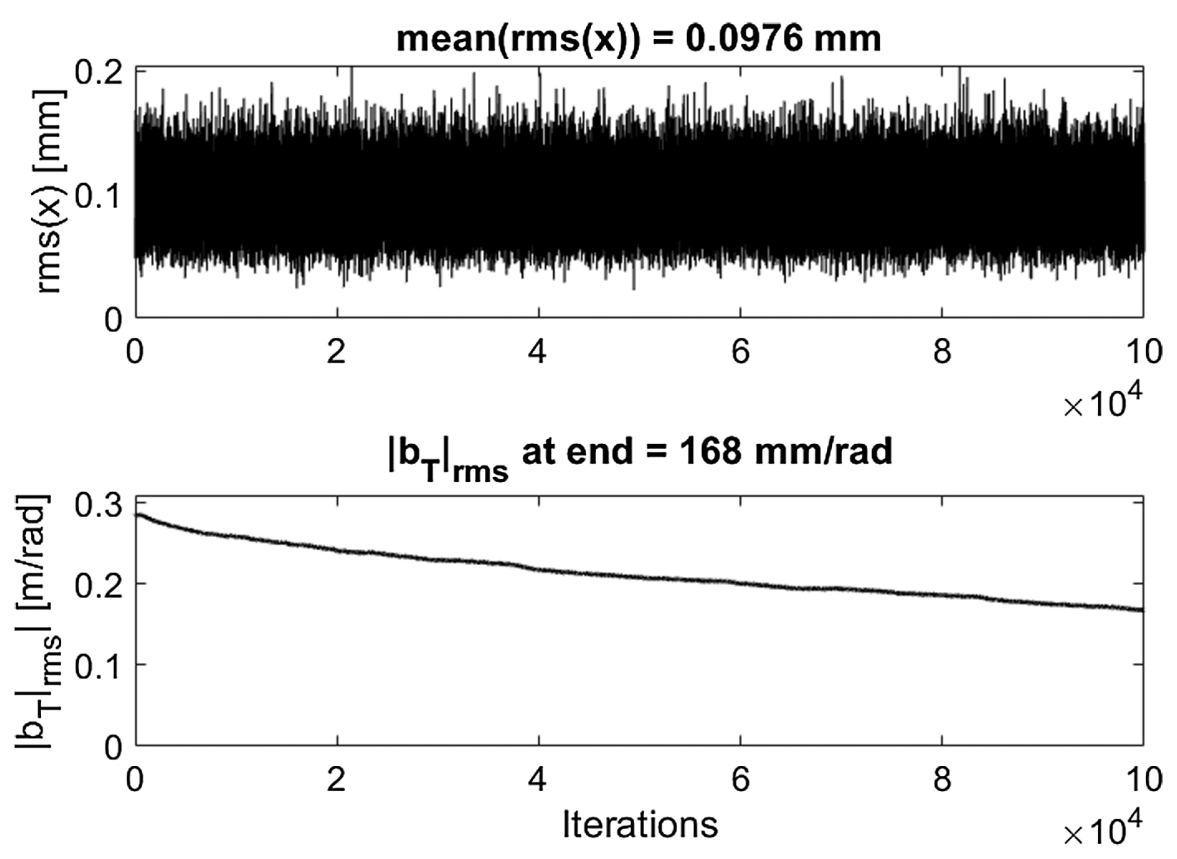

FIG. 2. The rms orbit $\sqrt{\langle x \mid x\rangle}$ (top) and the rms of all matrix elements of $B-\hat{B}$ (bottom) for $10^{5}$ iterations. The noise level was chosen $\sigma_{w}=0.1 \mathrm{~mm}$. The upper plot reproduces the noise level and the lower graph shows that the response-matrix estimate $\hat{B}$ slowly converges toward $B$. 


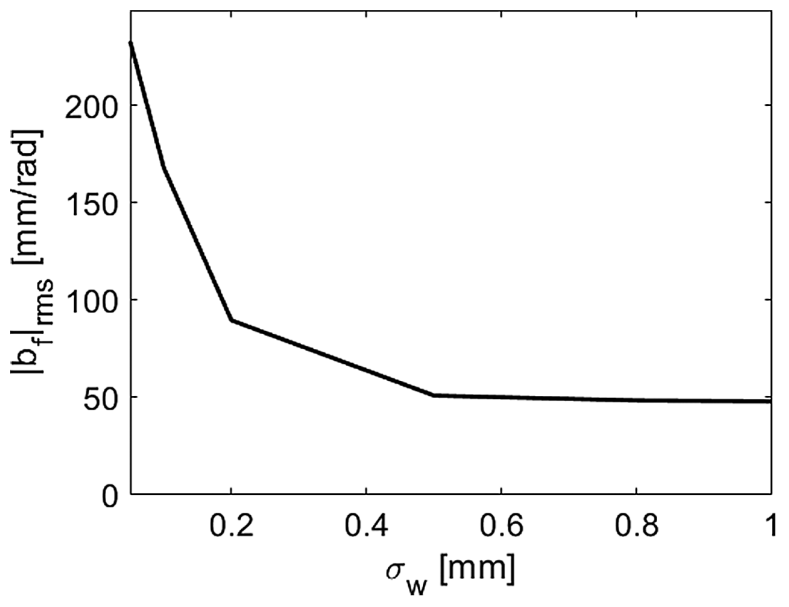

FIG. 3. The rms value of all matrix elements of $B-\hat{B}$ after $10^{5}$ iterations as a function of the noise level $\sigma_{w}$.

\section{DITHERING}

Varying the corrector excitations one at a time, either systematically or sinusoidally [15-17], in order to determine the response matrix is used in practically all accelerators. Moreover, continuously varying correctors very little such that the detrimental effect on the orbit is negligible, so-called dithering, was successfully used [18-20] to optimize the performance of a number of accelerators. We implement dithering in our simulation by adding a perturbing vector $\left|z_{t}\right\rangle$ to $\left|u_{t}\right\rangle$ when correcting the orbit in Eq. (2), which therefore becomes $\left|u_{t}\right\rangle=-K\left|x_{t}\right\rangle+\left|z_{t}\right\rangle$. The rest of the simulation remains unaffected; any changes of $\left|z_{t}\right\rangle$ and consequently of $\left|u_{t}\right\rangle$ are consistently accounted for in the updates of $P_{T}$ and $\hat{B}_{T}$ in Eqs. (6) and (7).
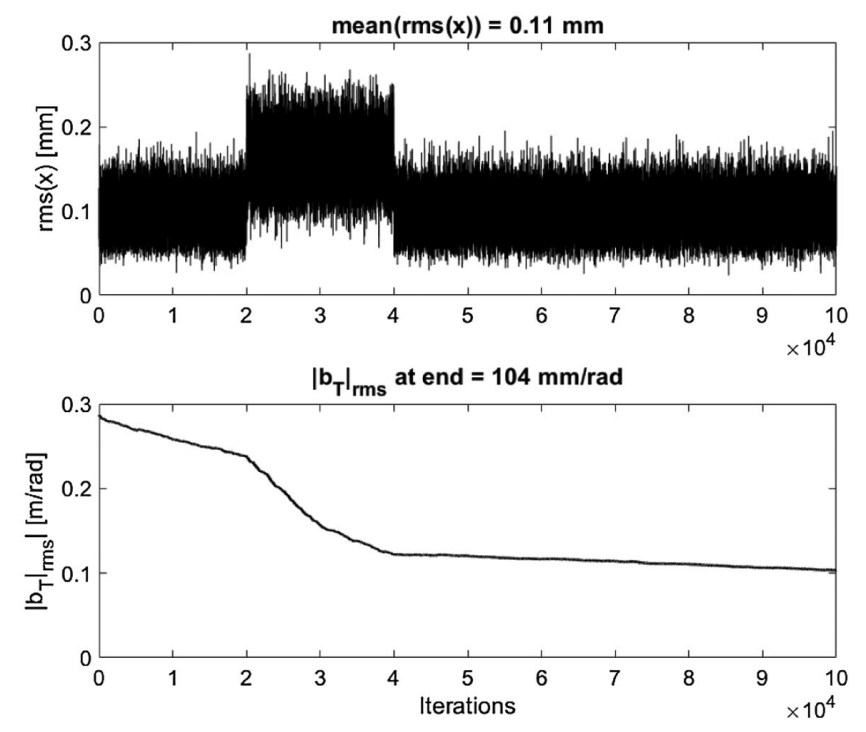

In the simulations, shown in Fig. 4, we chose to add $20 \mu \mathrm{rad}$ to the excitation $z^{k}$ to one corrector $k$ at a time in a round-robin fashion and record the rms orbit and the discrepancy for $10^{5}$ iterations. The plot on the left-hand side shows the simulation where the dithering was turned on between 20000 and 40000 iterations. We clearly see that the rms orbit increases from 0.10 to $0.16 \mathrm{~mm}$ during this period, which is consistent with expectations, because the rms value of the $B$ of $6.6 \mathrm{~m} / \mathrm{rad}$ and $20 \mu \mathrm{rad}$ additional excitation results in an additional rms orbit variation of $0.13 \mathrm{~mm}$, which, added in quadrature to $\sigma_{w}=0.1 \mathrm{~mm}$, gives about $0.16 \mathrm{~mm}$. We also observe on the lower plot that the discrepancy $\left|b_{T}\right|_{r m s}$ is significantly reduced and conclude that temporarily adding dithering helps to improve our knowledge of the response matrix. Note that no additional processing of the data is necessary. The algorithm learns whenever it gets the chance to observe some variation, never mind the source of the perturbation. Remarkably, a slammed door might be beneficial for something. In the simulation shown on the right-hand side in Fig. 4, we keep the $20 \mu \mathrm{rad}$ round-robin dithering on permanently and observe that the rms orbit is $0.16 \mathrm{~mm}$ throughout the simulation, while the discrepancy $\left|b_{T}\right|_{r m s}$ is reduced sevenfold. Again, no special processing is required.

The left-hand plot in Fig. 5 illustrates the effect of dither amplitude, shown on the horizontal axis, on the rms orbit (dashed black) and on the discrepancy (solid red). We clearly observe that the increasing dither amplitude increases the rms orbit $\sigma_{x}$, but at the same time, helps to reduce the discrepancy $\left|b_{f}\right|_{r m s}$. Closer inspection shows that a dither amplitude of $16 \mu \mathrm{rad}$ contributes to $\sigma_{x}$ with the same magnitude as normal noise level $\sigma_{w}$. This causes $\sigma_{x}$ to increase by $40 \%$. At the same time, $\left|b_{f}\right|_{r m s}$ is reduced by
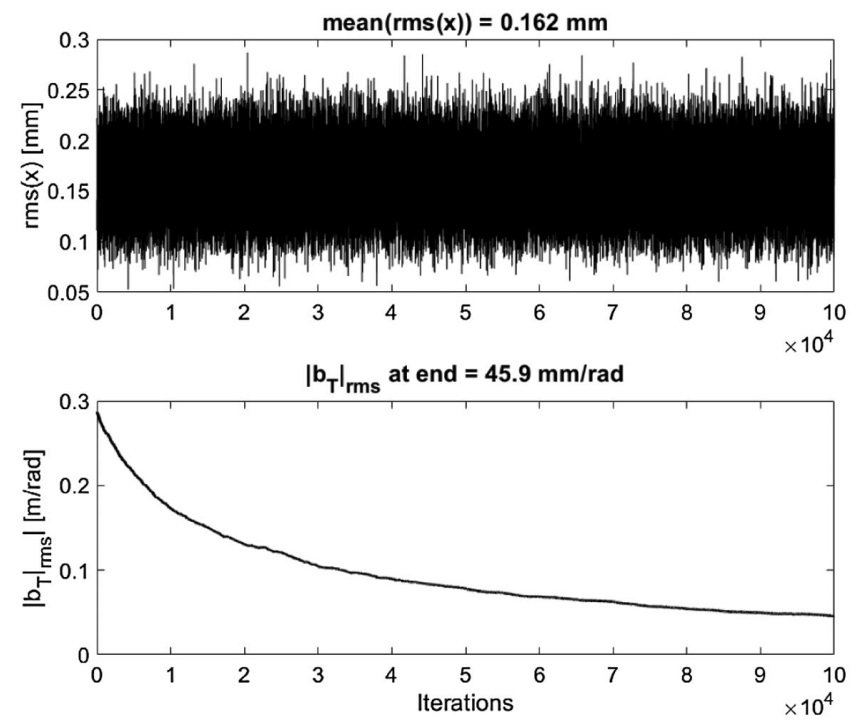

FIG. 4. Simulations based on parameters used for Fig. 2. Left: round-robin dithering with $20 \mu$ rad active from 20000 to 40000 iterations, which temporarily increases the rms orbit $\sigma_{x}$ but significantly helps to reduce the discrepancy $\left|b_{r m s}\right|$. Right: dithering with the same parameters is active all the time, which increases $\sigma_{x}$ all the time, but reduces $\left|b_{r m s}\right|$ even further. 

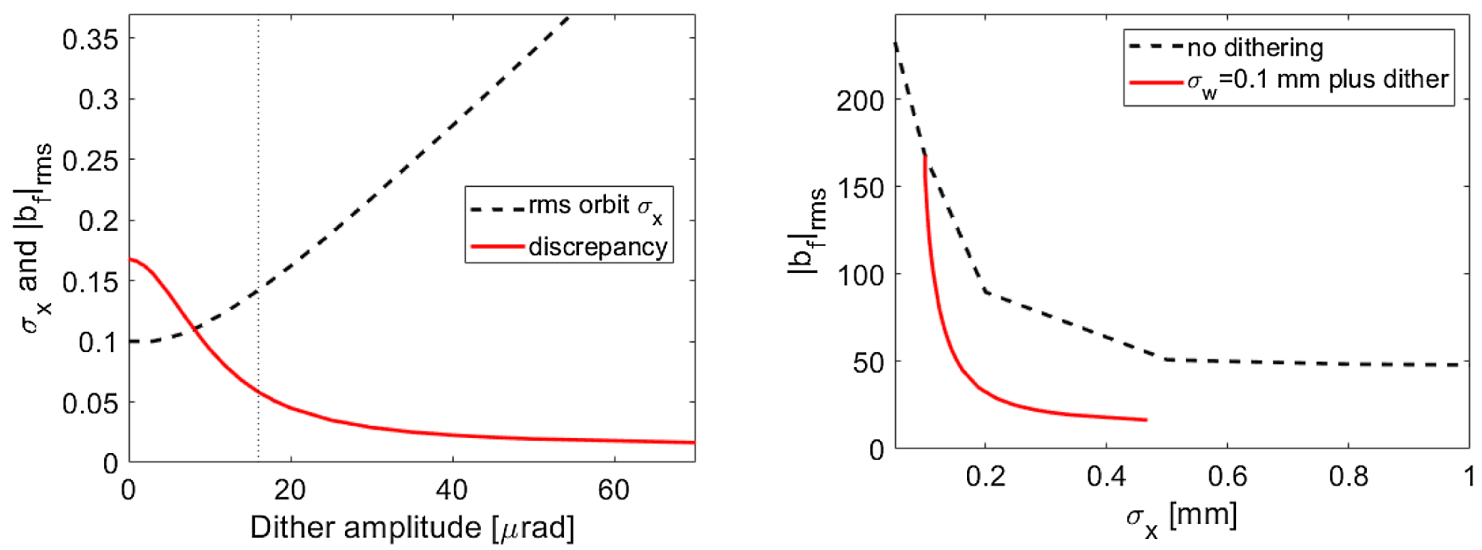

FIG. 5. Left: the rms orbit $\sigma_{x}$ (dashed black) and the discrepancy $\left|b_{r m s}\right|$ (solid red) as a function of the dither amplitude, which allows us to assess the trade-off between spoiling the orbit and learning the response matrix. Right: the solid red line shows $\left|b_{f}\right|_{r m s}$ plotted versus $\sigma_{x}$. The black dashed line shows the effect of purely random variations, already shown in Fig. 3, for comparison.

$1 / 3$ from $0.168 \mathrm{~m} / \mathrm{rad}$ to $0.056 \mathrm{~m} / \mathrm{rad}$. This configuration is indicated by the vertical dotted line in Fig. 5 .

The right-hand plot in Fig. 5 shows the data from the lefthand plot, but now plotting the discrepancy $\left|b_{f}\right|_{r m s}$ versus the rms orbit $\sigma_{x}$ (solid red) and compares it to the data from Fig. 3 (dashed black). Unsurprisingly, increasing $\sigma_{x}$ by dithering reduces $\left|b_{f}\right|_{r m s}$ more efficiently than just increasing the natural noise level $\sigma_{w}$.

\section{CONVERGENCE}

A matter of practical interest are the timescales, given by the number of iterations, before we observe some improvement of the response matrix. We point out that the results developed in the following sections apply to all systems described by Eq. (2), which includes rings with transverse coupling and correction matrices $K$ that use elaborate regularization schemes. The simulations, which are based on correctors and monitors in a single transverse plane, are only used to illustrate the general results. Let us start by analyzing the initial behavior of the discrepancy and approximate Eq. (6) by replacing $\left|u_{T+1}\right\rangle\left\langle u_{T+1}\right|$ by its expectation value $\mathbf{E}\left\{\left|u_{T+1}\right\rangle\left\langle u_{T+1}\right|\right\}$, which asymptotically becomes independent of $T$. We therefore use $\mathbf{E}\left\{\left|u_{T}\right\rangle\left\langle u_{T}\right|\right\}$ instead, which depends on $\left|x_{T}\right\rangle$ via $\left|u_{T}\right\rangle=-K\left|x_{T}\right\rangle$ and calculate

$$
\begin{aligned}
\left|x_{T}\right\rangle & =(1-B K)\left|x_{T-1}\right\rangle+\left|w_{T-1}\right\rangle \\
& =(1-B K)^{T}\left|x_{0}\right\rangle+\sum_{s=0}^{T-1}(1-B K)^{s}\left|w_{T-s-1}\right\rangle,
\end{aligned}
$$

where the second equality results from iterating the first equality. Since the spectral radius $\rho(\Lambda)$ with $\Lambda=1-B K$ is much less than unity, the influence of the initial $\left|x_{0}\right\rangle$ "dies out" for large $T$ and we can omit the first term from the sum. Inserting $\left|x_{T}\right\rangle$ in $\left|u_{T}\right\rangle=-K\left|x_{T}\right\rangle$, we obtain

$$
\begin{aligned}
\mathbf{E}\left\{\left|u_{T}\right\rangle\left\langle u_{T}\right|\right\} & =\mathbf{E}\left\{K\left[\sum_{s=0}^{T-1} \Lambda^{s}\left|w_{T-s-1}\right\rangle\right]\left[\sum_{r=0}^{T-1}\left\langle w_{T-r-1}\right|\left(\Lambda^{\top}\right)^{r}\right] K^{\top}\right\}+o(1) \\
& =K \sum_{s=0}^{T-1} \sum_{r=0}^{T-1} \Lambda^{s} \mathbf{E}\left\{\left|w_{T-s-1}\right\rangle\left\langle w_{T-r-1}\right|\right\}\left(\Lambda^{\top}\right)^{r} K^{\top}+o(1) \\
& =\sigma_{w}^{2} K \sum_{s=0}^{T-1}\left(\Lambda \Lambda^{\top}\right)^{s} K^{\top}+o(1),
\end{aligned}
$$

where we used that the expectation value of the Gaussian noise is $\mathbf{E}\left\{\left|w_{s}\right\rangle\left\langle w_{t}\right|\right\}=\sigma_{w}^{2} \delta_{s t} \mathbf{1}$. Moreover, $o(1)$ denotes a quantity that vanishes in the limit of large $T$. The smallness of $\rho(\Lambda)$ implies that only the term with $s=0$ in the sum in Eq. (10) contributes and we have $\mathbf{E}\left\{\left|u_{T}\right\rangle\left\langle u_{T}\right|\right\} \approx \sigma_{w}^{2} K K^{\top}$, which is indeed independent of $T$. We include round-robin dithering with amplitude $z$ through the $m$ correctors by adding a term $\frac{z^{2}}{m} \mathbf{1}$, because dithering is uncorrelated to the noise and after $m$ iterations dithering contributes a unit matrix. We thus just "spread out" this unit matrix to the individual iterations when diving by $m$. We therefore introduce 

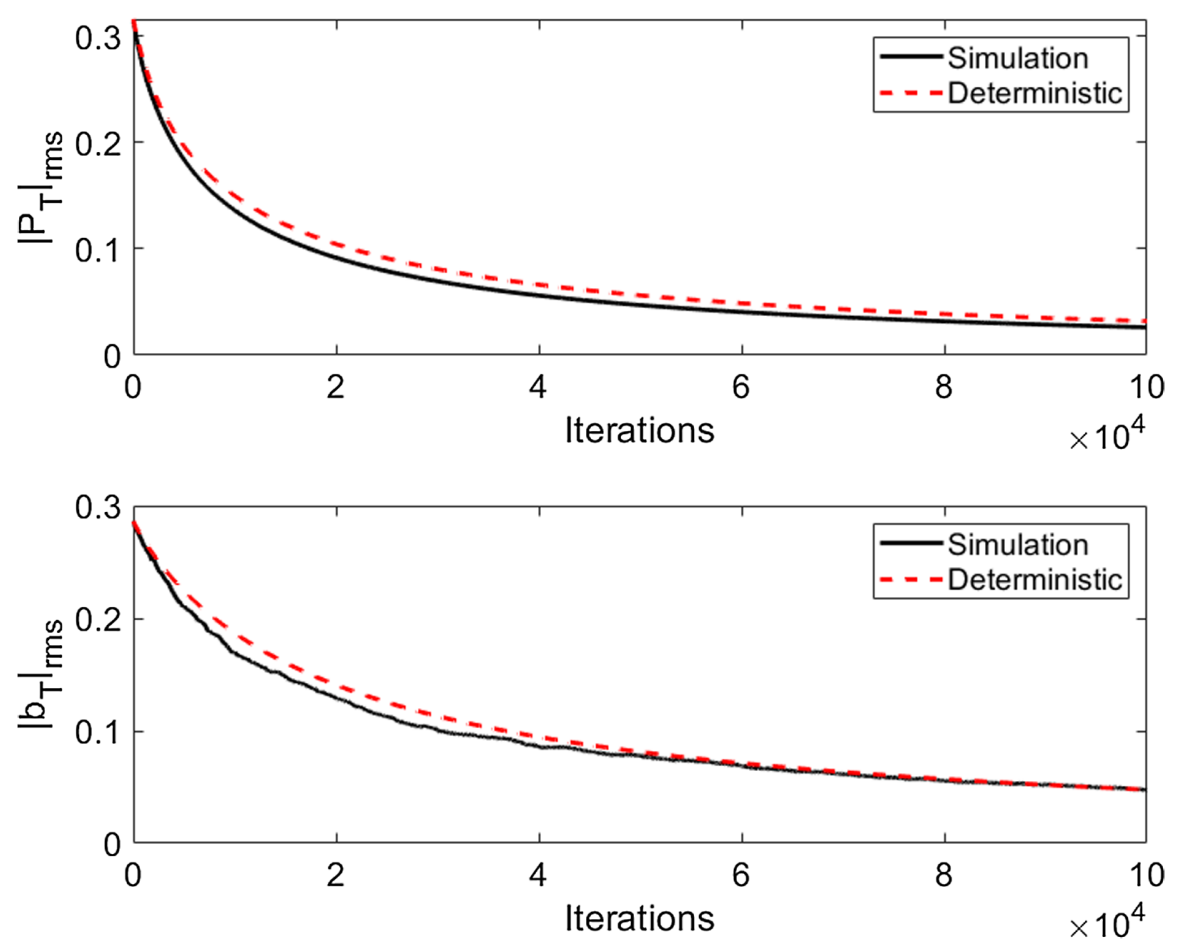

FIG. 6. The upper panel shows the evolution of $\left|P_{T}\right|_{2}$ (solid black) and $\left|\hat{P}_{T}\right|_{2}$ (dashed red) for a configuration with $\sigma_{w}=0.1 \mathrm{~mm}$ and $z=20 \mu \mathrm{rad}$. The lower panel shows the corresponding evolution of $b_{T}$ (solid black) and $\hat{b}_{T}$ (dashed red).

$$
Q=\sigma_{w}^{2} K K^{\top}+\frac{z^{2}}{m} \mathbf{1}
$$

to represent the average effect of the orbit correction and dithering when updating the "averaged" $\hat{P}_{T}$ in Eq. (6), which then reads

$$
\hat{P}_{T+1}=\hat{P}_{T}-\frac{\hat{P}_{T} Q \hat{P}_{T}}{1+\operatorname{Trace}\left(Q \hat{P}_{T}\right)} .
$$

Note that Eq. (12) is a deterministic equation that describes the averaged updating of $\hat{P}_{T}$. In the simulation we update $\hat{P}_{T}$ in parallel to its "stochastic brethren" $P_{T}$ and find that they are extremely close, both with and without dithering. The upper panel in Fig. 6 shows an example with $\sigma_{w}=$ $0.1 \mathrm{~mm}$ and $z=20 \mu \mathrm{rad}$, which corresponds to the configuration also displayed on the right-hand side in Fig. 4. The solid black curve is produced by a numerical simulation with simulated random noise and the dashed red curve shows the result of the deterministic simulation, based on Eqs. (11) and (12).

We point out that $Q$ is the only parameter in the dynamics described by Eq. (12). In order to simplify the analysis somewhat, we neglect the trace in the denominator, which is practically always much smaller than 1 , which results in $1+\operatorname{Trace}\left(Q \hat{P}_{T}\right) \approx 1$ and allows us to write the equation as $\hat{P}_{T+1}=\hat{P}_{T}-\hat{P}_{T} Q \hat{P}_{T}$. Moreover, $Q$ is symmetric by construction and we can choose a coordinate system in which $Q$ is diagonal with eigenvalues $\lambda_{j}=\sigma_{w}^{2} \sigma_{j}+z^{2} / m$, where $\sigma_{j}$ are the eigenvalues of $K K^{\top}$, such that $Q=O D O^{\top}$ with $D=\operatorname{diag}\left(\lambda_{1}, \ldots, \lambda_{m}\right)$ and an orthogonal matrix $O$. Also the starting guess for $P_{0}$ is the unit matrix and is diagonal, such that Eq. (12) can be written as $m$ independent equations for each of the $m$ diagonal elements $x_{j, T}$ of $\hat{P}_{T}$. Each eigenvalue thus corresponds to one mode that describes the dynamics of the convergence process. In the following, we consider one mode at a time and omit a second index $j=1 \ldots, m$ from $x$ and $\lambda$ to make the equations easier to read. We therefore obtain $x_{T+1}=x_{T}-\lambda x_{T}^{2}$ or its continuous approximation $d x_{T} / d T=-\lambda x_{T}^{2}$ for each mode. This equation has the solution

$$
x_{T}=\frac{x_{0}}{1+x_{0} \lambda T}
$$

Numerically $x_{0}$ has the value of unity, because $\hat{P}_{0}$ is the unit matrix, but we leave it in place to keep track of the units of $x_{0}$ which are $1 / \mathrm{mrad}^{2}$. We thus find that the inverse eigenvalues $1 / \lambda$ of the matrix $Q$ determine the timescales of the convergence of the process. Note, however, that the time dependence is inversely proportional to $T$, rather than exponential, and is therefore slow.

Figure 7 shows the timescales $1 / x_{0} \lambda_{j}$ with $j=1, \ldots, m$ for dither amplitudes $z$ between 0 and $30 \mathrm{mrad}$, while $\sigma_{w}$ is always $0.1 \mathrm{~mm}$. The dashed red line shows the timescale of the slowest mode and is given by the smallest eigenvalue 


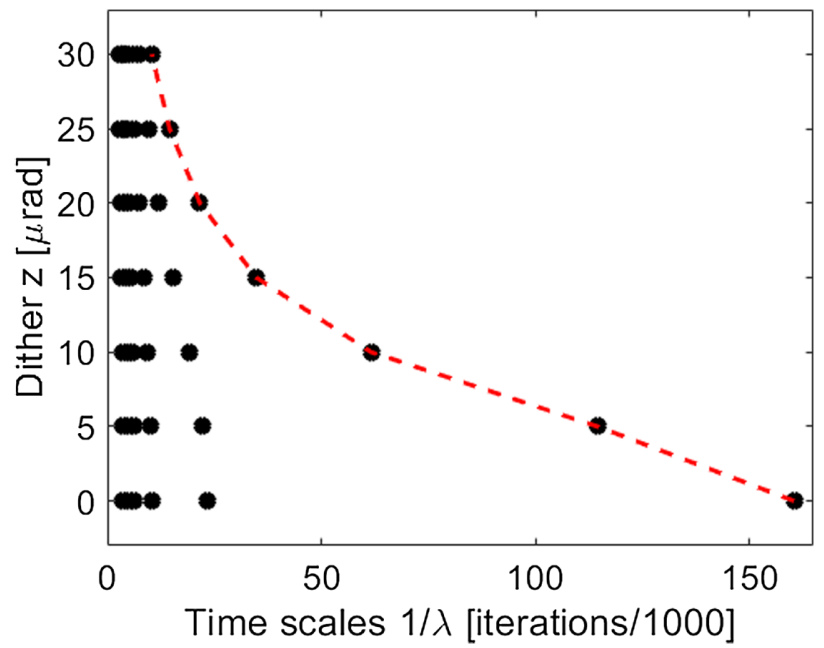

FIG. 7. Timescales of the convergence, determined from the inverse eigenvalues of $Q$ for dithering amplitudes $z$ from 0 to $30 \mathrm{mrad}$. In all cases we use $\sigma_{w}=0.1 \mathrm{~mm}$. The dashed red line shows the timescale $1 / \lambda_{\min }$ with $\lambda_{\min }=\sigma_{w}^{2} \sigma_{\min }\left[K K^{\top}\right]+z^{2} / m$ corresponding to the smallest eigenvalue of $Q$.

$\lambda_{\min }=\sigma_{w}^{2} \sigma_{\min }\left[K K^{\top}\right]+z^{2} / m$. Here $\sigma_{\min }[\cdot]$ is the smallest eigenvalue of the matrix in the argument. The rms orbit variation $\sigma_{x}$ approximately doubles in this range. We observe that there is always one very small eigenvalue, which leads to a very long timescale. Dithering mostly helps to reduce this long time scale from $1.6 \times 10^{5}$ to about 20000 iterations. At this point we remind ourselves, following the discussion from Sec. II, that $P_{T}=$ $\left(U_{T}^{\top} U_{T}\right)^{-1}$ determines the error bars of $\hat{B}_{T}$. Since Eq. (13) implies $P_{T} \propto 1 / T$ for $T \gtrsim 1 / x_{0} \lambda$ we find that the error bars of $\hat{B}_{T}$ have a $1 / \sqrt{T}$ dependence.

It remains to analyze the timescales of the convergence of the values of $\hat{B}_{T}$ to $B$, which is described by Eq. (7). We note that $\left|x_{T+2}\right\rangle-\left|x_{T+1}\right\rangle=B\left|u_{T+1}\right\rangle$ was caused by the corrector values $\left|u_{T+1}\right\rangle$, such that we arrive at

$$
\left(\hat{B}_{T+1}-B\right)=\left(\hat{B}_{T}-B\right)-\left(\hat{B}_{T}-B\right) \frac{\left|u_{T+1}\right\rangle\left\langle u_{T+1}\right| P_{T}}{1+\left\langle u_{T+1}\left|P_{T}\right| u_{T+1}\right\rangle},
$$

where we subtracted $B$ on both sides. We now replace $\left|u_{T+1}\right\rangle\left\langle u_{T+1}\right|$ by its expectation value and therefore use $Q$ from Eq. (11) to arrive at

$\hat{b}_{T+1}=\hat{b}_{T} \Xi_{T} \quad$ with $\quad \Xi_{T}=1-\frac{Q \hat{P}_{T}}{1+\operatorname{Trace}\left(Q \hat{P}_{T}\right)}$,

where we introduced $\hat{b}_{T}=\hat{B}_{T}-B$ to simplify the writing. Like Eq. (12) before is this a deterministic equation for $\hat{b}_{T}$ that we update in parallel to the stochastic simulations that generate $b_{T}$. On the bottom panel in Fig. 6 we show $\left|b_{T}\right|_{r m s}$, the rms value of $b_{T}$, as a solid black line and $\left|\hat{b}_{T}\right|_{\text {rms }}$ as dashed red line for a simulation with parameters specified in the figure caption. We take notice that both black and red curves track one another very well, which allows us to determine the timescales from analyzing $\Xi_{T}$ from Eq. (15). As before, we use a coordinate system in which $Q$ and $P_{T}$ are diagonal, ignore the denominator with the trace, and analyze one mode at a time. If we denote the eigenvalue of $\Xi_{T}$ by $\xi_{j}$ (and omit the index $\mathrm{j}$ henceforth, because we consider one mode at a time and want to use the subscript to denote the iteration), we find

$$
\xi_{T}=1-\lambda x_{T}=\frac{1-x_{0} \lambda+x_{0} \lambda T}{1+x_{0} \lambda T},
$$

where we substituted $x_{T}$ from Eq. (13). Again the timescales are determined by $1 / \lambda$, the inverse eigenvalues of $Q$. Inspecting Eq. (15), we see that the eigenvalues $\Xi_{s}$ describe how the modes decrease from one iteration $T-1$ to $T$. In order to find the total reduction after $T$ iterations we need to multiply all the previous eigenvalues $\xi_{s}$ for $1 \leq s \leq T$, which gives us the eigenvalues $y_{T}$ of the product $Y_{T}=\prod_{s=1}^{T} \Xi_{s}$

$$
y_{T}=\prod_{s=1}^{T} \xi_{s}=\prod_{s=1}^{T} \frac{1-x_{0} \lambda+x_{0} \lambda s}{1+x_{0} \lambda s}=\frac{1}{1+x_{0} \lambda T},
$$

where the last equality is straightforward to prove by induction. Thus $Y_{T}$ is a diagonal matrix with expressions $1 /\left(1+x_{0} \lambda T\right)$ along its diagonal. If we now rewrite this equation in nondiagonal coordinates, we obtain the matrix $G_{T}=O Y_{T} O^{\top}$ that maps the initial $\hat{b}_{0}$ to $\hat{b}_{T}$ after iteration $T$ via

$$
\begin{aligned}
\hat{b}_{T} & =\hat{b}_{0} G_{T} \quad \text { with } \\
G_{T} & =O \operatorname{diag}\left(\frac{1}{1+x_{0} \lambda_{1} T}, \ldots, \frac{1}{1+x_{0} \lambda_{m} T}\right) O^{\top}
\end{aligned}
$$

and $\lambda_{j}=\sigma_{w}^{2} \sigma_{j}\left[K K^{\top}\right]+z^{2} / m$ without iterating through all the intermediate steps. In passing, we point out that $G_{T}$ behaves like a transfer function that maps the initial $\hat{b}_{0}$ to a later value $\hat{b}_{T}$. Iterating with, for example, different dither amplitudes $z$ only involves left-multiplying with different $G_{T}$, each one calculated with the appropriate $z$.

Figure 8 shows several discrepancies $\left|b_{T}\right|_{r m s}$ as a function of the iteration number using double-logarithmic scales. On the left-hand plot we use a configuration with $\sigma_{w}=0.1 \mathrm{~mm}$ and no dithering. The black line shows $\left|b_{T}\right|_{\text {rms }}$ from the stochastic simulation, the red line shows $\left|\hat{b}_{T}\right|_{\text {rms }}$ using the deterministic iteration, while the blue dots are calculated with the matrix $G_{T}$. We observe that all three curves track one another very well. The plot on the righthand side in Fig. 8 shows the configuration with $20 \mu \mathrm{rad}$ dithering added, already used in Fig. 6 with the blue dots from the analytic calculation superimposed. Again, the 

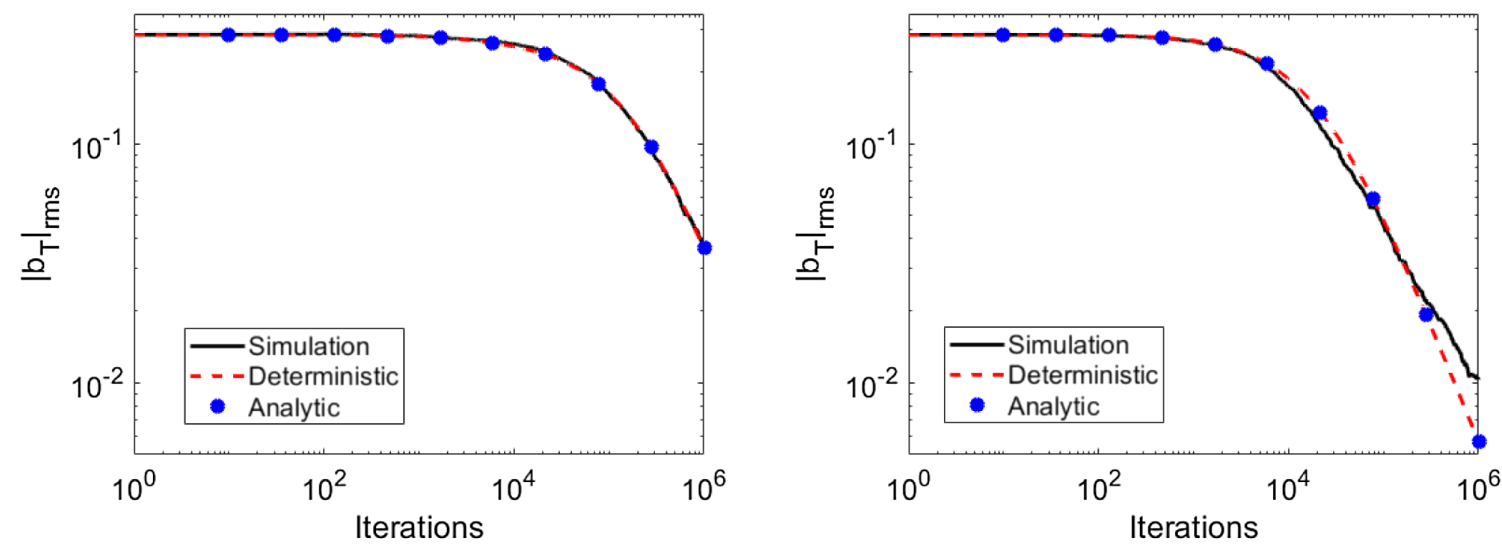

FIG. 8. Left: the discrepancies $\left|b_{T}\right|_{r m s}$ calculated by direct numerical simulation (black line), from Eq. (12) (red dashes), and from Eq. (18) (blue dots) for $\sigma_{w}=0.1 \mathrm{~mm}$ and no dither. Right: with $20 \mu \mathrm{rad}$ round-robin dither added.

agreement is rather good, though some discrepancies show up, once $\left|b_{T}\right|_{r m s}$ becomes very small. Let us therefore analyze this late regime more carefully.

From the discussion in Sec. II we know that $2 \sigma_{w}^{2} P_{T}=$ $2 \sigma_{w}^{2}\left(U_{T}^{\top} U_{T}\right)^{-1}$ is a data-driven approximation of the covariance matrix for the matrix elements of $\hat{B}_{T}$. We therefore heuristically approximate the error bars by $\sigma(\hat{B})=\sqrt{2\left|P_{T}\right|_{r m s}} \sigma_{w}$ and show $\left|b_{T}\right|_{r m s}$ for a numerical simulation (solid black) and the deterministic average (red dashes) as well as $\sigma(\hat{B})$ (blue dash-dots) in Fig. 9. We observe that once $\left|b_{T}\right|_{r m s}$ becomes smaller than $\sigma(\hat{B})$ the numerical simulation significantly differs from the averaged model. In this regime the approximations, in particular, factoring the expectation value of the product of $\hat{B}_{T}-B$ and $\left|u_{T+1}\right\rangle\left\langle u_{T+1}\right| P_{T}$ into separate expectation values no longer hold. Here, the statistical fluctuations around the mean and the $1 / \sqrt{T}$ scaling of the error bars (blue dash-dots) become the dominating factor for the rate of

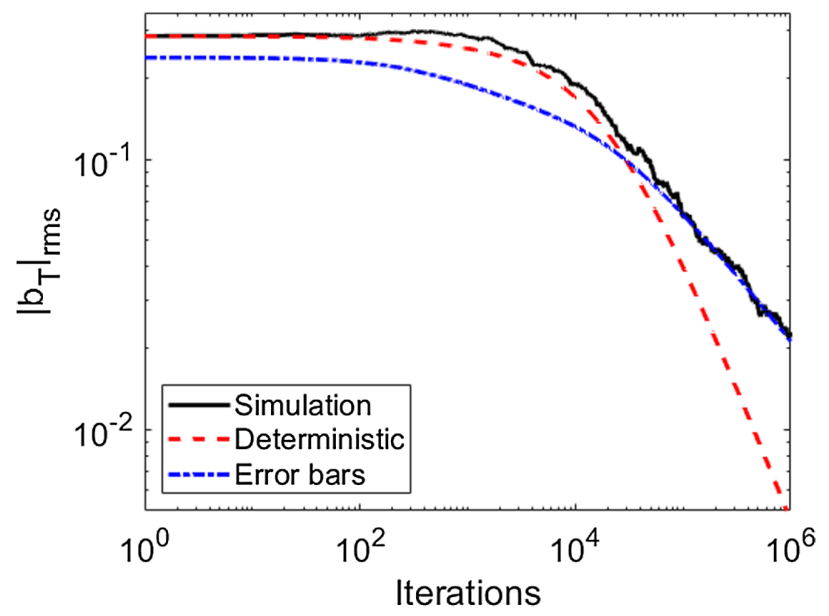

FIG. 9. The rms value of the discrepancy $\left|b_{T}\right|_{r m s}$ from a numerical simulation (solid black) and from iterating Eqs. (12) and (15) as well as the approximate error bars $\sigma(\hat{B})$ (blue dashes) for a configuration with $\sigma_{w}$ increased to $0.3 \mathrm{~mm}$ and no dither. convergence. We therefore need to address the asymptotic regime separately, which is the topic of the next section.

\section{ASYMPTOTICS}

The asymptotic regime is characterized by the discrepancy $\left|b_{T}\right|_{r m s}$ being smaller than the error bars, or heuristically; the signal $\left|b_{T}\right|_{r m s}$ is inside the noise floor. We saw in the simulations shown on the figures that even in this regime $\hat{B}_{T}$ converges toward the real response matrix $B$. If we focus on cases without dithering $(z=0)$, we can explore this further by exploiting a theorem by Lai and Wei [21], which states that

$$
\left|\hat{B}_{T}-B\right|_{\infty}=O\left(\sqrt{\frac{\log \left(\sigma_{\max }\left[P_{T}^{-1}\right]\right)}{\sigma_{\min }\left[P_{T}^{-1}\right]}}\right),
$$

where $\sigma_{\min }[\cdot]$ and $\sigma_{\max }[\cdot]$ denote the smallest and largest eigenvalue of the matrix in the argument, respectively. $|\cdot|_{\infty}$ denotes the largest value of the matrix in the argument, which is always larger than the rms value of all matrix elements that we used in the previous sections; the two values only differ by a numerical factor of order unity. The symbol $O(\cdot)$ denotes the leading order in $T$, and $P_{T}^{-1}=$ $U_{T}^{\top} U_{T}=\sum_{t=1}^{T}\left|u_{t}\right\rangle\left\langle u_{t}\right|$ was defined earlier. We therefore need to determine the scaling of $\sum_{t=1}^{T}\left|u_{t}\right\rangle\left\langle u_{t}\right|$ and its smallest and largest eigenvalues with $T$.

To do so, we note that the system, defined by Eq. (2), can be written as $\left|x_{t+1}\right\rangle=(1-B K)\left|x_{t}\right\rangle+\left|w_{t}\right\rangle$, which shows that the time step $t+1$ only depends on parameters at time $t$, which makes it a Markov chain. Moreover, if the closedloop system is stable, the spectral radius $\rho(\Lambda)$, with $\Lambda=1-B K$, is strictly less than unity, which causes the process to forget all uniformly bounded initial conditions sufficiently fast. This makes the corresponding Markov chain uniformly ergodic and implies that the time-average and the average over the distribution function of the noise, the expectation value $\mathbf{E}\{\cdot\}$, are the same 


$$
\frac{1}{T} \sum_{t=1}^{T}\left|u_{t}\right\rangle\left\langle u_{t}\right|=\mathbf{E}\left\{\left|u_{T}\right\rangle\left\langle u_{T}\right|\right\}+o(1)
$$

where, as before, $o(1)$ is an expression that vanishes in the limit of large $T$. The right-hand side of Eq. (20) we already calculated in Eq. (10) and turn to its asymptotic behavior, which is encapsulated in the limit of $\Gamma_{T}=\sum_{s=0}^{T-1}\left(\Lambda \Lambda^{\top}\right)^{s}$ for large $T$. First we note that

$$
\sum_{s=0}^{T-1}\left(\Lambda \Lambda^{\top}\right)^{s} \leq \sum_{s=0}^{\infty} \rho(\Lambda)^{2 s} \mathbf{1}=\frac{1}{1-\rho(\Lambda)^{2}} \mathbf{1}<\infty
$$

is finite. Second, the existence can be proven by noting that $\Gamma_{T}$ is a Cauchy sequence; $\Gamma_{T}-\Gamma_{T^{\prime}}=o(1)$ for large $T, T^{\prime}$. We can therefore introduce $\Gamma=\lim _{T \rightarrow \infty} \Gamma_{T}$ and obtain

$$
\mathbf{E}\left\{\left|u_{T}\right\rangle\left\langle u_{T}\right|\right\}=\sigma_{w}^{2} K \Gamma K^{\top}+o(1) .
$$

This expression allows us to determine the smallest and largest eigenvalue of the left-hand side

$$
\sigma_{\min }\left[\mathbf{E}\left\{\left|u_{T}\right\rangle\left\langle u_{T}\right|\right\}\right]=\sigma_{w}^{2} \sigma_{\min }\left[K \Gamma K^{\top}\right]+o(1)
$$

and likewise for $\sigma_{\max }$. We note that the smallest and largest eigenvalues of a matrix are continuous functions of the matrix elements. This implies - as a consequence of the continuous mapping theorem [22] - that limits of these functions are preserved, even if the matrix elements depend on random variables. We therefore obtain from Eq. (20)

$$
\begin{aligned}
\sigma_{\min }\left[\sum_{t=1}^{T}\left|u_{t}\right\rangle\left\langle u_{t}\right|\right] & =\sigma_{\min }\left[T \mathbf{E}\left\{\left|u_{T}\right\rangle\left\langle u_{T}\right|\right\}+o(T)\right] \\
& =\sigma_{w}^{2} T \sigma_{\min }\left[K \Gamma K^{\top}\right]+o(T)
\end{aligned}
$$

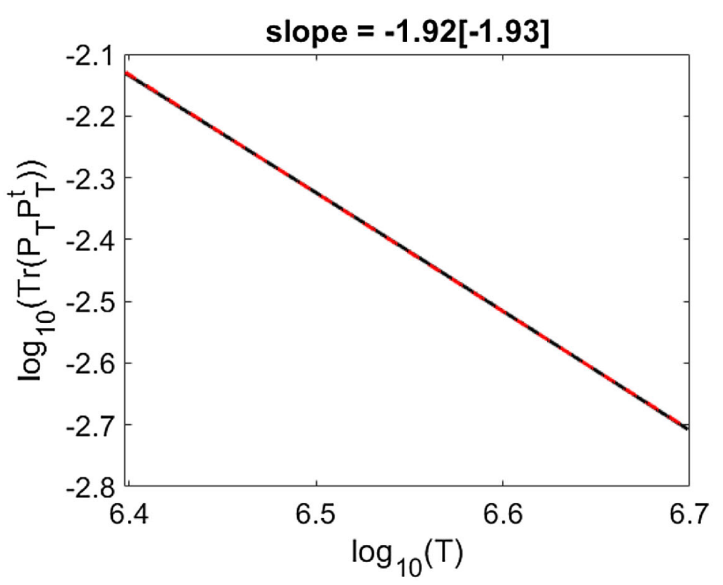

and likewise for $\sigma_{\max }$. Here $o(T)$ denotes a quantity that increases strictly slower with $T$ than $T$. Moreover, the convergence of the random variables on the left-hand side toward the expectation value on the right-hand side happens with probability 1 -almost surely in the mathematical literature. Summarily, both $\sigma_{\min }\left[P_{T}^{-1}\right]$ and $\sigma_{\max }\left[P_{T}^{-1}\right]$ asymptotically scale linearly with $T$.

For the asymptotic approach of the estimate $\hat{B}_{T}$ toward the real response matrix $B$ we insert the eigenvalues in Eq. (19) and find

$$
\left|\hat{B}_{T}-B\right|_{\infty}=O\left(\sqrt{\frac{\log T}{T \sigma_{\min }\left[K \Gamma K^{\top}\right]}}\right),
$$

where we did not spell out constant factors. In passing we note that $P_{T}=\left(U_{T}^{\top} U_{T}\right)^{-1}$ scales with $1 / \sigma_{\min }\left[P_{T}^{-1}\right]$ and this leads to

$$
P_{T}=O\left(\frac{1}{\sigma_{w}^{2} T \sigma_{\min }\left[K \Gamma K^{\top}\right]}\right)
$$

which decreases like $1 / T$ in the leading order.

In order to verify the asymptotics numerically we run simulations with $\sigma_{w}=0.1 \mathrm{~mm}$ for $5 \times 10^{6}$ iterations. Figure 10 shows the asymptotic behavior of Trace $\left(P_{T}^{\top} P_{T}\right)$ and of $\left|b_{T}\right|_{r m s}$ as a function of the iteration number on a double logarithmic scale in the range between 2.5 and $5 \times 10^{6}$ iterations. A linear fit to the data on the left-hand side shows a slope of -1.92 , if fitting the entire range, and -1.93 , if fitting the upper $20 \%$. This indicates an approximate tendency toward Trace $P_{T}^{\top} P_{T} \propto 1 / T^{2}$, which is consistent with Eq. (26). Repeating these calculations for different random seeds gives comparable results. On the other hand, the slope of $\left|b_{T}\right|_{r m s}$ is approximately 0.57 , which is close to

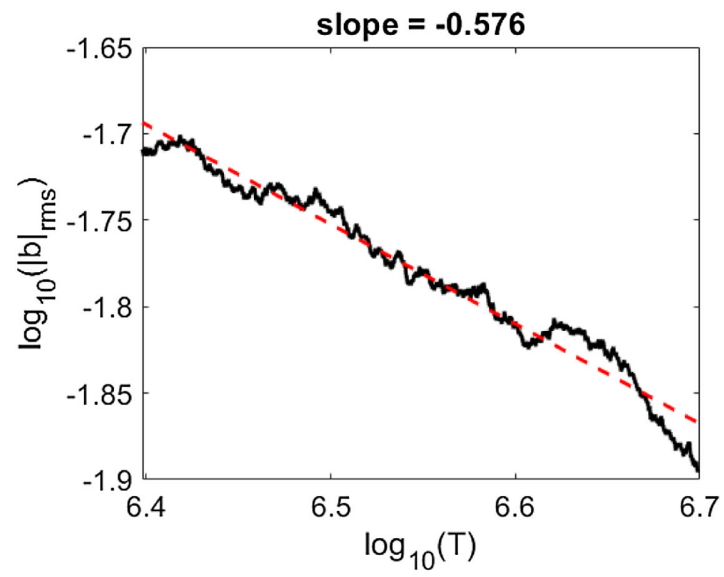

FIG. 10. Left: plotting the logarithm Trace $\left(P_{T}^{\top} P_{T}\right)$ versus the log of the number of iterations for $\sigma_{w}=0.1 \mathrm{~mm}$ shows that the slope is close to -2. Right: in the same way plotting the log of $\left|b_{T}\right|_{r m s}$ shows a slope of approximately $\kappa=-0.57$. The red dashes denote the fitted straight lines. 

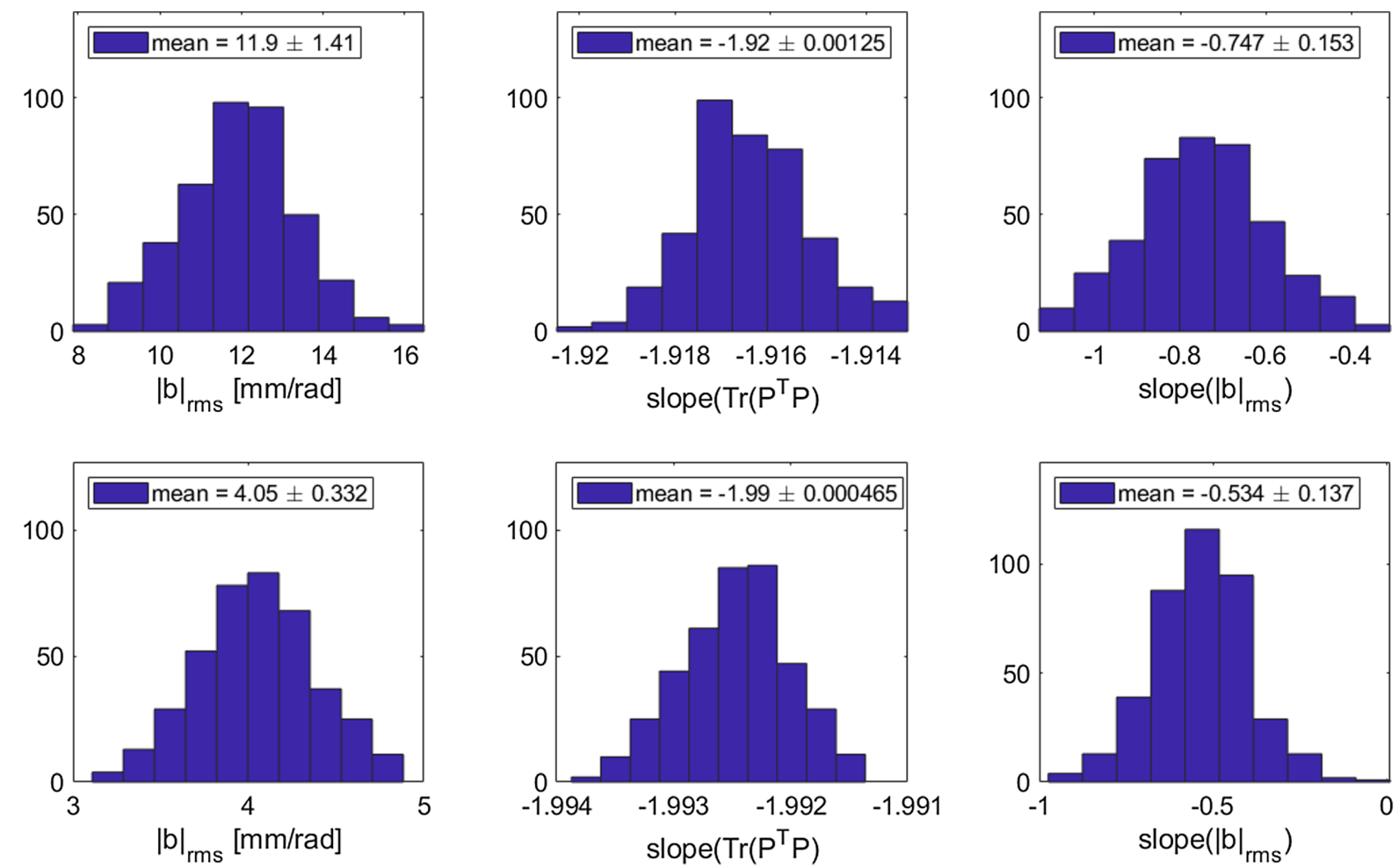

FIG. 11. Top row: histograms of the final discrepancy $\left|b_{f}\right|_{r m s}$ after $5 \times 10^{6}$ iterations (left) with $\sigma_{w}=0.1 \mathrm{~mm}$, the slope of Trace $\left(P_{T}^{\top} P_{T}\right)$ (center) and the slope of $\left|b_{T}\right|_{r m s}$ (right) in the range $2.5 \times 10^{6}$ to $5 \times 10^{6}$ iterations. Bottom row: the corresponding plots with $20 \mu \mathrm{rad}$ round-robin dither added.

$1 / \sqrt{T}$, the dominant dependence in Eq. (25). But the curve is much more noisy, which we attribute to the logarithm of $T$ in the numerator of Eq. (25).

In order to explore this variability we run the simulation with 400 different random seeds, all having $\sigma_{w}=0.1 \mathrm{~mm}$, and plot the final value of the discrepancy $\left|b_{f}\right|_{r m s}$, the slope of Trace $\left(P_{T}^{\top} P_{T}\right)$, and the slope of $\left|b_{T}\right|_{r m s}$ in the top row of histograms in Fig. 11. We see that after $5 \times 10^{6}$ iterations $\left|b_{f}\right|_{r m s}$ has reached a value of about $12 \mathrm{~mm} / \mathrm{rad}$ (left). The slope of Trace $\left(P_{T}^{\top} P_{T}\right)$ is -1.92 (center) and has not quite reached its asymptotic value of -2 . The asymptotic slope of $\left|b_{T}\right|_{r m s}$ (right) is approximately 0.75 . The width of the histograms indicate their standard deviations, which is indicated as the uncertainty in the respective legends of the plots. We observe that the results are reasonably stable and give a good indication of the asymptotic behavior of the system. In the bottom row in Fig. 11 we show the corresponding plots for the situation, where $20 \mu \mathrm{rad}$ round-robin dither is added. We find that the final value of $\left|b_{f}\right|_{r m s}$ is only $4 \mathrm{~mm} / \mathrm{rad}$ (left), while the slope of Trace $\left(P^{\top} P\right)$ is very close to the asymptotic value of -2 . The slope of the discrepancy $\left|b_{T}\right|_{\text {rms }}$ (right) indicates a value of approximately -0.53 . We point out that the width of the two histograms on the right is much larger than the others, which we again attribute to the logarithm in the numerator of Equation (25).

\section{SOME TECHNICAL ASPECTS}

We now turn to practical aspects of our system to determine the real response matrix $B$. From Eqs. (25) and (26) we see that the most important quantity for convergence is the smallest eigenvalue of $K \Gamma K^{\top}$, where $\Gamma=\sum_{s=0}^{\infty}\left(\Lambda \Lambda^{\top}\right)^{s} \quad$ is defined immediately before Equation (22). For all well-behaved feedback systems $\rho(\Lambda)=\rho(1-B K)$ is much smaller than unity and the term with $s=0$ dominates the sum, which makes $\Gamma$ very close to the $m \times m$ unit matrix. Since we do not a priori know $B$, we just set $\Gamma$ to the unit matrix when evaluating the performance of our system and consider $K K^{\top}$ alone.

If the feedback system is equipped with more correctors than position monitors $(n<m)$, the matrix $K K^{\top}$ is degenerate and has a null eigenvalue, which spoils the convergence. The left-hand plot in Fig. 12 shows what happens when we remove one row, corresponding to one position monitor, from the response matrix and repeat the analysis. The orbit, shown on the upper panel is still corrected with a rms value comparable to $\sigma_{w}$, but $\left|b_{T}\right|_{r m s}$, shown on the lower panel, no longer converges to zero. The identification of the response matrix only works partially and a finite difference to the real $B$ remains.

If, on the other hand, there are more position monitors than corrector magnets $(n>m)$-in the simulation we removed one column, corresponding to one corrector 

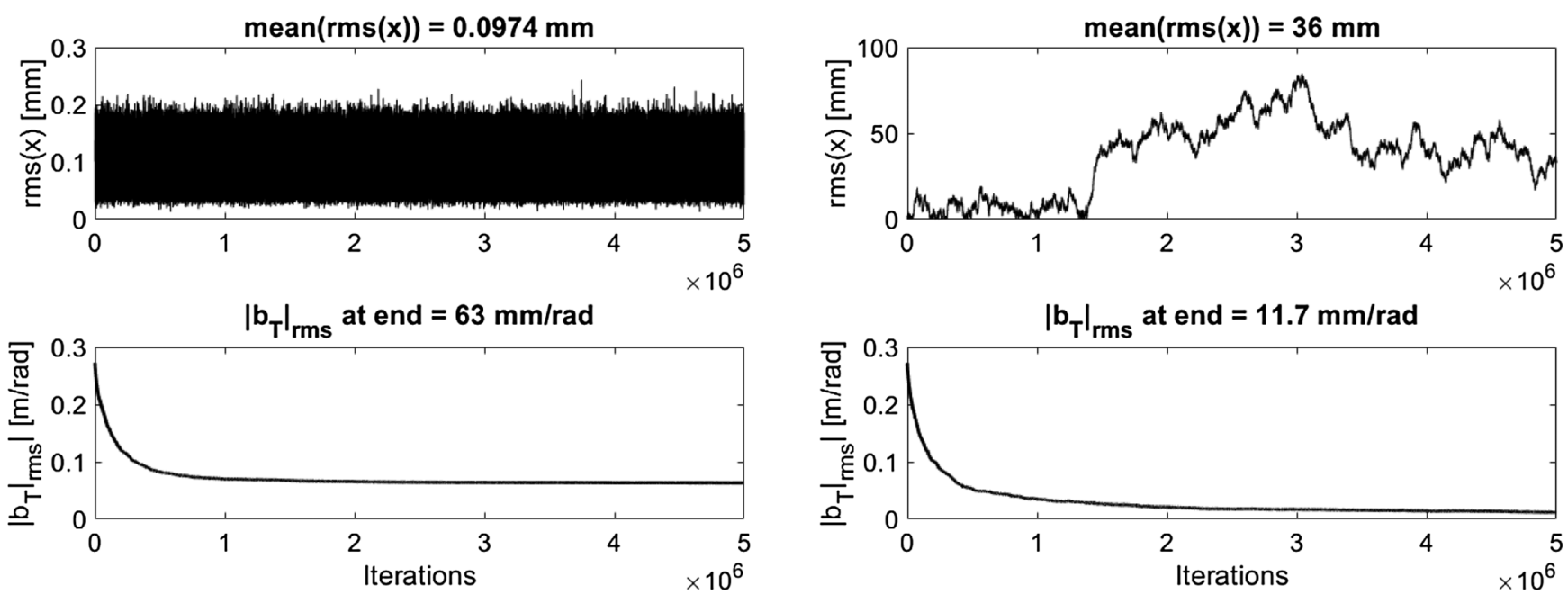

FIG. 12. Left: one position monitor is removed $(n<m)$, which spoils the convergence of $\left|b_{T}\right|_{r m s}$ to zero, but maintains a small orbit. Right: one corrector magnet is removed $(n>m)$; now the identification of the response matrix works well and $\left|b_{T}\right|_{r m s}$ converges to zero, but the orbit is not corrected properly.

magnet, from the response matrix - the identification of the response matrix works well, as illustrated on the lower panel on the right-hand plot in Figure 12, because $m \times m$ matrix $K K^{\top}$ has full rank-no null eigenvalues. On the other hand, we can no longer correct the orbit, as shown on the upper panel, because now the $m \times n$ matrix $K$ now has eigenvalues null. We can, however, remedy this problem by decomposing the symmetric $n \times n$ matrix $K^{\top} K=O D O^{\top}$, where $D$ is a diagonal matrix containing the eigenvalues $d_{i}$ and $O$ is an orthogonal matrix, whose columns are the corresponding eigenvectors $\left|o_{i}\right\rangle$. We note that $\Phi=$ $\sum_{i \text { in nullspace }}\left|o_{i}\right\rangle\left\langle o_{i}\right|$ is a projection matrix onto the nullspace of $K^{\top} K$, such that $\Psi=1-\Phi$ projects onto its orthogonal complement, which is the space of BPM readings that the correctors can actually affect. If we use $\Psi|x\rangle$ instead of $|x\rangle$ when we apply the correction, the null-modes never pile up and become unstable. If we apply this method to the example from the right-hand side in Fig. 12, the orbit in the upper panel looks very similar to the one on the lefthand plot. Since we always know $K$ (as opposed to $B$, which we do not know), we can always construct $\Psi$. Using the projector $\Psi$ we can also use our algorithm if there are more BPM than correctors.

For one-to-one orbit correction feedback systems with equal number of position monitors and correctors $(n=m)$ we just have to evaluate the eigenvalues of $K K^{\top}$ and possibly adjust $K$ by hand in order to speed up the convergence, albeit at the expense of compromising the orbit correction to some extent. The details depend on the particular accelerator and we will not dwell on this point further.

In order to understand the scaling of the convergence with system parameters, we consider rings with increasing number of $n=m$ cells with equal phase advance that contain one corrector and one BPM, each, which results in a near-circulant response matrix [23]. In numerical experiments we find that the largest eigenvalue of $B^{\top} B$ approximately increases with $n^{2}$. Since the correction matrix $K$ is normally close to the pseudoinverse of $B$, we expect the smallest eigenvalue of $K K^{\top}$ to have an inverse dependence on $n^{2}$. Moreover, $B$ is proportional to a typical value of the beta function $\hat{\beta}$ in the ring, which makes $K \propto 1 / \hat{\beta}$, such that we find $\sigma_{\min }\left[K K^{\top}\right] \propto\left(\sigma_{w} / n \hat{\beta}\right)^{2}$; the algorithm works best in small rings with noisy BPM.

It is instructive to compare the achievable error bars for the response matrix with those of an open loop measurement campaign, which are approximately given by $\sigma(B)_{o} \approx \sigma_{w} / \hat{\theta}$, where $\hat{\theta}$ is the amplitude of the corrector excitations. In Sec. II we found that error bars of $\hat{B}$ from the closed-loop measurements are given by $\sigma(\hat{B})^{2}=$ $\operatorname{diag}\left[2 \sigma_{w}^{2}\left(U_{T} U_{T}^{\top}\right)^{-1}\right]=2 \sigma_{w} \operatorname{diag}\left[P_{T}\right]$. Moreover, during the early stages of the convergence, the eigenvalues of $P_{T}$ are given by Eq. (13). We see that all eigenvalues decrease with $x_{0} /\left(1+x_{0} \lambda_{i} T\right)$, albeit at a slow timescale, characterized by the eigenvalues $\lambda_{i}$ of $Q$. This process continues until the asymptotic regime is reached, as discussed near the start of Sec. VI. In the asymptotic regime $P_{T}$ continues to decrease as specified by Eq. (26). We conclude that the error bars always get smaller and do so without limit. Additionally, Eq. (25) implies that the approximation $\hat{B}$ asymptotically approaches the "true" response matrix $B$.

Finally, extending the algorithm to include settling time $\tau$, processing delay $d$, and relaxation into a new equilibrium with timescale $\tau_{d}$ is straightforward by introducing unobservable state variables $\left|\alpha_{t}\right\rangle$ and $\left|\beta_{t}\right\rangle$. Their dynamic behavior is described by 


$$
\begin{aligned}
& \left|\alpha_{t}\right\rangle=\frac{\tau}{\tau+1}\left|\alpha_{t-1}\right\rangle+\frac{1}{\tau+1}\left|u_{t-1-d}\right\rangle \quad \text { and } \\
& \left|\beta_{t}\right\rangle=\frac{\tau_{d}}{\tau_{d}+1}\left|\beta_{t-1}\right\rangle+\frac{1}{\tau_{d}+1} B\left|\alpha_{t}\right\rangle
\end{aligned}
$$

with $\left|u_{t-1-d}\right\rangle=-K\left|x_{t-1-d}\right\rangle$. The delay $d$ and time constants $\tau$ and $\tau_{d}$ affect the stability of the closed-loop system, but we assume that the feedback designer has chosen $K$ to ensure its stability. In the equation, $\left|\alpha_{t}\right\rangle$ corresponds to the field inside the vacuum chamber that the beam actually "sees" and $\left|\beta_{t}\right\rangle$, for example, the damping due to synchrotron radiation. The observable beam position $\left|x_{t}\right\rangle$ then updates with $\left|x_{t+1}\right\rangle=\left|x_{t}\right\rangle+\left|\beta_{t}\right\rangle$. We note that the left side of Eq. (27) enables us to uniquely determine the $\left|\alpha_{t}\right\rangle$ from the $\left|u_{t}\right\rangle$, which makes them quasi observable, provided $\tau$ and $d$ are known. Moreover, we find the $\left|\beta_{t}\right\rangle=\left|x_{t+1}\right\rangle-$ $\left|x_{t}\right\rangle$ from the $\left|x_{t}\right\rangle$, which turns the right side of Eq. (27) into $\left(\tau_{d}+1\right)\left|\beta_{t}\right\rangle-\tau_{d}\left|\beta_{t-1}\right\rangle=B\left|\alpha_{t}\right\rangle$. We observe that this equation has the same form as Eq. (3) from the main text with one component of the left-hand side taking the place of $x_{s+1}^{i}-x_{s}^{i}$ shifted by one time step. Likewise, $\left|\alpha_{t}\right\rangle$ takes the place of $\left|u_{s}\right\rangle$. The analysis from this report up to Eqs. (6) and (7) remains valid, but analyzing the convergence and the asymptotics goes beyond the scope of the present report.

\section{CONCLUSIONS}

We applied standard system identification techniques, based on recursive least-squares methods, to determine the response matrix in parallel to correcting the orbit in a storage ring. Simulations show that the method works well, though it is rather slow and requires a large number of iterations. The speed can, however, be increased significantly by systematically adding small perturbations to the corrector magnets, so-called dithering. In this way a small deterioration of the orbit quality can be balanced with the desire to determine an accurate response matrix. We found that the convergence of $\hat{B}_{T}$ to the "real" response matrix is governed by the eigenvalues $\lambda$ of the matrix $Q$ from
Eq. (11) and we solved the time dependence of the discrepancy $\hat{b}_{T}=\hat{B}_{T}-B$ with some approximations. We found in Eq. (18) that $\hat{b}_{T}$ scales with $1 / T$, but only until the magnitude of $\hat{b}_{T}$ becomes smaller than the error bars of the fitting process, which scale with $1 / \sqrt{T}$. Once inside the noise level, we found that the asymptotic behavior of the convergence has a $\sqrt{\log (T) / T}$ dependence and is governed by the smallest eigenvalue of $K K^{\top}$. In particular, both the error bars of the approximation $\hat{B}_{T}$ and the difference between $\hat{B}_{T}$ and the real $B$ tend to zero in the limit of large $T$. Furthermore, we found that those feedback systems with number of BPMs equal or larger than the number of correctors $(n \geq m)$ permit us to simultaneously stabilize the orbit and to identify the response matrix $B$.

Several extensions of this work come to mind. First, optimizing the correction matrix $K$ such that the smallest eigenvalue of $K K^{\top}$ is as large as possible without spoiling the orbit quality $\sigma_{x}$. Second, comparing different correction strategies, for example, deriving $K$ from "optimal control" quality measures that put a weight both on the orbit $\sigma_{x}^{2}$ and the rms corrector excitation. Third, finding an optimal strategy to make the dither amplitude $z$ time-varying, such that global measure of performance that balances orbit correction and system identification is minimized. The regret, studied for instance in [24], may serve as an example.

\section{ACKNOWLEDGMENTS}

This work was supported in part by the Swedish Research Council (grant No. 2016-00861), and the Swedish Foundation for Strategic Research (Project CLAS).

\section{APPENDIX A: SHERMAN-MORRISON FORMULA}

Here we show that $P_{T+1}$ is given by Eq. (6) if its inverse $P_{T+1}^{-1}$ is given by $P_{T+1}^{-1}=P_{T}^{-1}+\left|u_{T+1}\right\rangle\left\langle u_{T+1}\right|$. To show this, we explicitly calculate $P_{T+1}^{-1} P_{T+1}$ and show that it evaluates to the unit matrix

$$
\begin{aligned}
P_{T+1}^{-1} P_{T+1} & =\left[P_{T}^{-1}+\left|u_{T+1}\right\rangle\left\langle u_{T+1}\right|\right]\left[P_{T}-\frac{P_{T}\left|u_{T+1}\right\rangle\left\langle u_{T+1}\right| P_{T}}{1+\left\langle u_{T+1}\left|P_{T}\right| u_{T+1}\right\rangle}\right] \\
& =P_{T}^{-1} P_{T}+\left|u_{T+1}\right\rangle\left\langle u_{T+1}\right| P_{T}-\frac{P_{T}^{-1} P_{T}\left|u_{T+1}\right\rangle\left\langle u_{T+1}\left|P_{T}+\right| u_{T+1}\right\rangle\left\langle u_{T+1}\left|P_{T}\right| u_{T+1}\right\rangle\left\langle u_{T+1}\right| P_{T}}{1+\left\langle u_{T+1}\left|P_{T}\right| u_{T+1}\right\rangle} \\
& =1+\left|u_{T+1}\right\rangle\left\langle u_{T+1}\right| P_{T}-\frac{\left|u_{T+1}\right\rangle\left(1+\left\langle u_{T+1}\left|P_{T}\right| u_{T+1}\right\rangle\right)\left\langle u_{T+1}\right| P_{T}}{1+\left\langle u_{T+1}\left|P_{T}\right| u_{T+1}\right\rangle} \\
& =1
\end{aligned}
$$

and we can use Eq. (6) to update $P_{T}$ with the new information that is encoded in the new corrector excitations $|\# 1\rangle u_{T+1}$. Note that $P_{T}=\left(U_{T}^{\top} U_{T}\right)^{-1}$ and its inverse are symmetric by construction for all $T$. This implies that the order of multiplication of $P_{T+1}$ and its inverse does not matter and we also have $P_{T+1} P_{T+1}^{-1}=1$. 


\section{APPENDIX B: RESPONSE-MATRIX UPDATE}

Here we follow [13] and show that the update of the response matrix $\hat{B}$ is accomplished by Eq. (7). We therefore write Eq. (5) for time step $T+1$

$$
\begin{aligned}
\left|\hat{B}_{T+1}^{i:}\right\rangle & =\left(U_{T+1}^{\top} U_{T+1}\right)^{-1} U_{T+1}^{\top}\left(\begin{array}{c}
y_{1}^{i} \\
\vdots \\
y_{T+1}^{i}
\end{array}\right)=P_{T+1}\left[\sum_{s=1}^{T} y_{s}^{i}\left|u_{s}\right\rangle+y_{T+1}^{i}\left|u_{T+1}\right\rangle\right] \\
& =\left[P_{T}-\frac{P_{T}\left|u_{T+1}\right\rangle\left\langle u_{T+1}\right| P_{T}}{1+\left\langle u_{T+1}\left|P_{T}\right| u_{T+1}\right\rangle}\right]\left[\sum_{s=1}^{T} y_{s}^{i}\left|u_{s}\right\rangle+y_{T+1}^{i}\left|u_{T+1}\right\rangle\right] .
\end{aligned}
$$

Here we introduce the abbreviation $y_{T}^{i}=x_{T+1}^{i}-x_{T}^{i}$, exploit that $U_{T+1}^{\top}=\left(\left|u_{1}\right\rangle, \ldots,\left|u_{T+1}\right\rangle\right)$, and finally express $P_{T+1}$ through Eq. (6). In the next step we multiply the two square brackets and obtain four terms

$$
\begin{aligned}
\left|\hat{B}_{T+1}^{i:}\right\rangle= & P_{T} \sum_{s=1}^{T} y_{s}^{i}\left|u_{s}\right\rangle+P_{T} y_{T+1}^{i}\left|u_{T+1}\right\rangle-\frac{P_{T}\left|u_{T+1}\right\rangle\left\langle u_{T+1}\left|P_{T} \sum_{s=1}^{T} y_{s}^{i}\right| u_{s}\right\rangle}{1+\left\langle u_{T+1}\left|P_{T}\right| u_{T+1}\right\rangle} \\
& -\frac{P_{T}\left|u_{T+1}\right\rangle\left\langle u_{T+1}\left|P_{T}\right| u_{T+1}\right\rangle y_{T+1}^{i}}{1+\left\langle u_{T+1}\left|P_{T}\right| u_{T+1}\right\rangle} \\
= & \left|\hat{B}_{T}^{i:}\right\rangle+P_{T} y_{T+1}^{i}\left|u_{T+1}\right\rangle-\frac{P_{T}\left|u_{T+1}\right\rangle\left\langle u_{T+1} \mid \hat{B}_{T}^{i:}\right\rangle}{1+\left\langle u_{T+1}\left|P_{T}\right| u_{T+1}\right\rangle}-y_{T+1}^{i} \frac{P_{T}\left|u_{T+1}\right\rangle\left\langle u_{T+1}\left|P_{T}\right| u_{T+1}\right\rangle}{1+\left\langle u_{T+1}\left|P_{T}\right| u_{T+1}\right\rangle}
\end{aligned}
$$

where, according to Eq. (5), we identify the estimate in the previous iteration $T$ as $\left|\hat{B}_{T}^{i}\right\rangle=P_{T} \sum_{s=1}^{T}\left|u_{s}\right\rangle y_{s}^{i}$. Combining the second and the fourth term, we arrive at

$$
\left|\hat{B}_{T+1}^{i:}\right\rangle=\left|\hat{B}_{T}^{i:}\right\rangle+\left[x_{T+2}^{i}-x_{T+1}^{i}-\left\langle u_{T+1} \mid \hat{B}_{T}^{i:}\right\rangle\right] \frac{P_{T}\left|u_{T+1}\right\rangle}{1+\left\langle u_{T+1}\left|P_{T}\right| u_{T+1}\right\rangle} .
$$

Taking the transpose of this equation and stacking the rows on top of each other then leads to Eq. (7).

\section{APPENDIX C: CODE FOR ONE ITERATION}

The following function receives $\hat{B}$ and $P$, as well as the recently measured orbit $|x\rangle$ and the dither vector $|z\rangle$ as input and returns the updated matrices $\hat{B}_{\text {new }}$ and $P_{\text {new }}$ as well as the orbit $\left|x_{\text {new }}\right\rangle$ after the correction is applied. Inside the function, first the externally defined noise level $\sigma_{w}$, a constant correction matrix $\tilde{B}$, the real response matrix $B$, and the correction matrix $K$ are supplied as global variables. Next, using $K$, the new corrector values $|u\rangle$ are calculated, dither $|z\rangle$ is added to the correctors, and the new orbit $\left|x_{\text {new }}\right\rangle$ is calculated, including the noise $|w\rangle$, here implemented as normally distributed random numbers. Then the auxiliary quantity $\left\langle u_{T+1}\right| P_{T}$ is stored in the variable tmp and the inverse of the denominator in the last term in Eq. (6) is calculated. The next two lines are straight implementations of Eqs. (6) and (7).

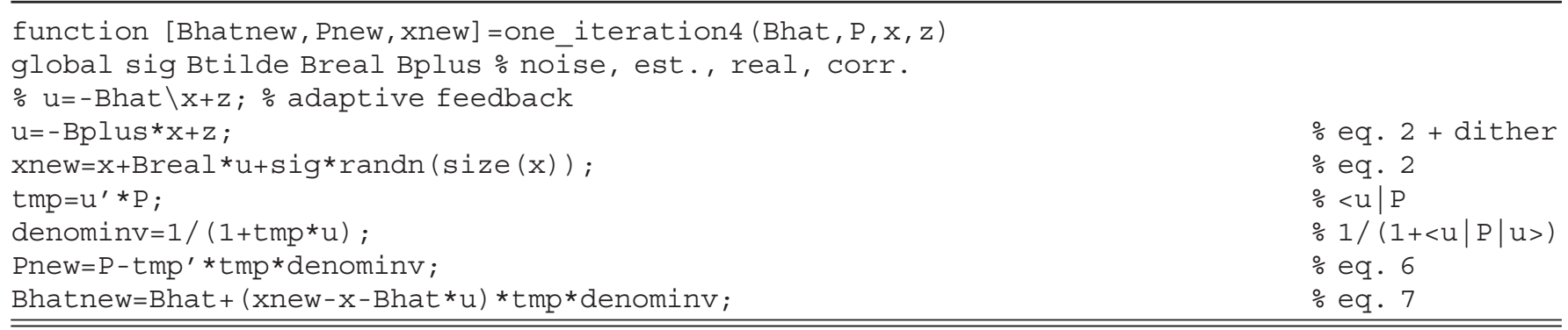


The figures in the main body of the report are produced by iterating this function. Note that in the above code the correction matrix $K$ is fixed. We can, however, easily make the feedback adaptive by simply replacing this line in the code by $u=-B h a t \backslash x+z$, as indicated in the commentedout line. In this way, always the most recent approximation for the matrix $\hat{B}_{T}$ is used when correcting the orbit.

\section{APPENDIX D: SPATIALLY CORRELATED MONITOR NOISE}

The assumption that the noise $\left|w_{t}\right\rangle$ of position monitors is uncorrelated, is easily relaxed and in this Appendix we show how spatially correlated noise, characterized by $\mathbf{E}\left\{\left|v_{t}\right\rangle\left\langle v_{s}\right|\right\}=\sigma_{w}^{2} \delta_{t s} C$ affects the rest of the results, where $\sigma_{w}^{2} C$ is the covariance matrix of the noise. Its matrix elements on the diagonal $\sigma_{w}^{2} C_{i i}$ describe the square of the error bars of BPM $i$ and the off-diagonal elements describe the correlations among different BPMs. Note that we separated the magnitude of the noise $\left(\sigma_{w}^{2}\right)$ from the correlations, where $C$ is a positive definite and symmetric matrix with matrix elements of order unity.

Since $C$ is symmetric we can decompose it into orthogonal matrices $O$ and a diagonal matrix. Since it is positive definite, all its eigenvalues are positive and we can write the diagonal matrix as the square of another diagonal matrix $D$

$$
C=O D^{2} O^{\top} .
$$

We will now use this representation of $C$ to transform the dynamical system represented by Eq. (2), but with correlated noise $\left|v_{t}\right\rangle$

$$
\left|x_{t+1}\right\rangle=\left|x_{t}\right\rangle+B\left|u_{t}\right\rangle+\left|v_{t}\right\rangle
$$

and multiply it with $D^{-1} O^{\top}$ from the left, which results in

$$
D^{-1} O^{\top}\left|x_{t+1}\right\rangle=D^{-1} O^{\top}\left|x_{t}\right\rangle+D^{-1} O^{\top} B\left|u_{t}\right\rangle+D^{-1} O^{\top}\left|v_{t}\right\rangle .
$$

With the transformed variables

$\left|y_{t}\right\rangle=D^{-1} O^{\top}\left|x_{t}\right\rangle, \quad B^{\prime}=D^{-1} O^{\top} B, \quad\left|w_{t}\right\rangle=D^{-1} O^{\top}\left|v_{t}\right\rangle$.

Equation (D3) reads

$$
\left|y_{t+1}\right\rangle=\left|y_{t}\right\rangle+B^{\prime}\left|u_{t}\right\rangle+\left|w_{t}\right\rangle,
$$

where we have

$$
\begin{aligned}
\mathbf{E}\left\{\left|w_{t}\right\rangle\left\langle w_{s}\right|\right\} & =D^{-1} O^{\top} \mathbf{E}\left\{\left|v_{t}\right\rangle\left\langle v_{s}\right|\right\} O D^{-1} \\
& =D^{-1} O^{\top} \sigma_{w}^{2} C \delta_{t s} O D^{-1}=\sigma_{w}^{2} \delta_{t s} \mathbf{1}
\end{aligned}
$$

We find that this system is equivalent to the one from Eq. (2), such that we can directly use the methods developed in the main body of this report. We only need to undo the transformation from Eq. (D4) in the end.

If we apply this procedure to Eq. (6) and (7) we find that these equations are unchanged; the improvement of the $\hat{B}$ does not depend on the noise as long as there are perturbations. Only the changes of the controller $\left|u_{t}\right\rangle$ and the resulting orbit changes matter.

The correlation matrix $C$ does, however, affect the convergence of the algorithm. Using correlated noise $\left|v_{t}\right\rangle$ instead of $\left|w_{t}\right\rangle$ in Eq. (10), we find that its last equality becomes

$$
\mathbf{E}\left\{\left|u_{T}\right\rangle\left\langle u_{T}\right|\right\}=\sigma_{w}^{2} K \sum_{s=0}^{T-1} \Lambda^{s} C\left(\Lambda^{\top}\right)^{s} K^{\top}+o(1) .
$$

Following the reasoning from the main body, the term with $s=0$ is dominant, which gives us $\mathbf{E}\left\{\left|u_{T}\right\rangle\left\langle u_{T}\right|\right\}=$ $\sigma_{w}^{2} K C K^{\top}$ and the matrix $Q$ from Eq. (11) becomes $Q=\sigma_{w}^{2} K C K^{\top}+\frac{z^{2}}{m}$. With this version of $Q$ the conclusions of Sec. V remain the same.

Also the asymptotic behavior is affected by $C$. Equation (21) becomes

$$
\begin{aligned}
\Gamma_{T} & =\sum_{s=0}^{T-1} \Lambda^{s} C\left(\Lambda^{\top}\right)^{s} \leq \sum_{s=0}^{\infty} \rho(C) \rho(\Lambda)^{2 s} \mathbf{1} \\
& =\frac{\rho(C)}{1-\rho(\Lambda)^{2}} \mathbf{1}<\infty
\end{aligned}
$$

where $\rho(C)$ is the spectral radius of $C$. This results in a slight redefinition of $\Gamma=\lim _{T \rightarrow \infty} \Gamma_{T}$ which still is finite, which renders the remainder of the Sec. VI valid.

[1] J. P. Koutchouk, Trajectory and closed orbit correction, in Frontiers of particle beams, Springer Lecture Notes in Physics 343, 46 (1989).

[2] M. Minty and F. Zimmermann, Measurement and Control of Charged Particle Beams (Springer, Heidelberg, 2003).

[3] X. Huang, Beam-based Correction and Optimization for Accelerators (CRC Press, Boca Raton, 2020).

[4] V. Ziemann, Imperfections and correction, contribution to the CERN Accelerator School: Introduction to Accelerator Physics, https://arxiv.org/abs/2006.11016, 2020.

[5] G. Rehm, Characterization of closed orbit feedback systems, Proceedings of the eighth beam instrumentation conference IBIC2019 in Malmö, 2019 (JACoW Publishing, Geneva, Switzerland, 2019), p. 479, https://accelconf.web .cern.ch/ibic2019/papers/weco01.pdf.

[6] S. H. Mirza, R. Singh, P. Forck, and B. Lorentz, Performance of the closed orbit feedback systems with spatial model mismatch, Phys. Rev. Accel. Beams 23, 072801 (2020). 
[7] J. Corbett, M. Lee, and V. Ziemann, A fast model calibration procedure for storage rings, in Proceedings of the Particle Accelerator Conference PAC93, Washington, 1993 (IEEE, New York, 1993), p. 108.

[8] J. Safranek, Experimental determination of storage ring optics using orbit response measurements, Nucl. Instrum. Methods Phys. Res., Sect. A 388, 27 (1997).

[9] W. Corbett, J. Safranek, D. Robin, and V. Ziemann, Debugging real accelerators, Part. Accel. 58, 193 (1997), http://cds.cern.ch/record/325028/files/p193.pdf.

[10] G. Goodwin and R. Payne, Dynamic System Identification (Academic Press, London, 1977).

[11] L. Ljung, System Identification; Theory for the User, 2nd ed. (Prentice Hall, New Jersey, 1999).

[12] W. Press et al., Numerical Recipes, 2nd ed. (Cambridge University Press, Cambridge, 1992).

[13] Section VII.2 in [10].

[14] V. Ziemann, Hands-on Accelerator Physics Using Matlab (CRC Press, Boca Raton, 2019).

[15] I. Martin et al., A fast optics correction for the diamond storage ring, in the International Particle Accelerator Conference IPAC2014 (JACoW, Geneva, 2014), p. 1763.

[16] X. Yang, V. Smaluk, L. H. Yu, Y. Tian, and K. Ha, Fast and precise technique for magnet lattice correction via sinewave excitation of fast correctors, Phys. Rev. Accel. Beams 20, 054001 (2017).
[17] Z. Marti et al., Fast orbit response matrix measurements at ALBA, in the International Particle Accelerator Conference IPAC2017 (JACoW, Geneva, 2017), p. 365.

[18] M. Ross, L. Hendricksen, T. Himel, and E. Miller, Precise system stabilization at SLC using dither techniques, Report No. SLAC-PUB-6102, 1993.

[19] S. Gierman et al., New fast dither system for PEP-II, Report No. SLAC-PUB-12679.

[20] M. Masuzawa et al., Early commissioning of the luminosity dither system for SuperKEKB, presented at the Seventh International Beam Instrumentation Conference (IBIC18) in Shanghai, China (JACoW Publishing, Geneva, Switzerland, 2018).

[21] T. Lai and C. Wei, Least squares estimates in stochastic regression models with applications to identification and control of dynamic systems, Ann. Stat. 10, 143 (1982).

[22] Van der Waart, Asymptotic Statistics (Cambridge University Press, Cambridge, England, 1998); see Theorem 2.3.

[23] S. Mirza, R. Singh, P. Forck, and H. Klingbeil, Closed orbit correction at synchrotrons for symmetric and nearsymmetric lattices, Phys. Rev. Accel. Beams 22, 072804 (2019).

[24] I. Ziemann and H. Sandberg, On uninformative optimal policies in adaptive LQR with unknown B-matrix, arXiv: 2011.09288 . 